\title{
氧化偶联反应的最新研究进展
}

\author{
张 剑 ${ }^{a}$ 陆庆全 ${ }^{a}$ 刘 超 ${ }^{a}$ 雷爱文*,a,b \\ ( ${ }^{a}$ 武汉大学化学与分子科学学院 高等研究院 武汉 430027) \\ ( $b$ 江西师范大学国家单糖化学合成工程技术研究中心 南昌 330022)
}

\begin{abstract}
摘要 近年来, 过渡金属催化的氧化偶联反应已成为有机合成化学中构建碳一碳键以及各类碳一杂键的重要方法. 主 要综述了我们研究小组在氧化偶联领域, 尤其是第三代氧化偶联反应方面所取得的研究进展, 介绍了各类反应的特 点、优势及在合成中的应用.

关键词 氧化偶联; 氧化岸基化; 氧化酯化; 亲核试剂
\end{abstract}

\section{Recent Advances in Oxidative Coupling Reactions}

\author{
Zhang, $\operatorname{Jian}^{a}$ \\ Lu, Qingquan ${ }^{a}$ \\ Liu, Chao ${ }^{a}$ \\ Lei, Aiwen*,a,b \\ $\left({ }^{a}\right.$ College of Chemistry and Molecular Sciences, Institute for Advanced Studies (IAS), Wuhan University, Wuhan 430072) \\ ( ${ }^{b}$ National Research Center for Carbohydrate Synthesis, Jiangxi Normal University, Nanchang 330022)
}

\begin{abstract}
Recently, transition-metal-catalyzed oxidative coupling reactions have emerged as the most important and attractive methods to construct carbon-carbon bond and carbon-heteroatom bonds in organic synthetic chemistry. This review mainly summarizes our research progress in the area of oxidative coupling and their versatility, application in organic synthesis, especially for the third generation of oxidative coupling.

Keywords oxidative coupling; oxidative carbonylation; oxidative esterification; nuclephiles
\end{abstract}

偶联反应始于 20 世纪 60 年代, 是各类亲电试剂在 金属催化剂催化下与各种金属亲核试剂形成碳一碳键 以及各类碳一杂键的反应. 这类经典偶联反应在底物的 普适性与官能团兼容性等方面有着优异表现, 自发现至 今得到了快速发展, 在过去几十年当中一直都是有机合 成研究的热点, 其研究成果广泛应用于生物、医药、农 药、仿生、材料、天然产物全合成等各个领域当中, 极 大地促进了有机化学发展 ${ }^{[1]}$. 该领域也涌现出了一批杰 出的化学家, 成就了众多的人名反应 ${ }^{[2]}$, 正是由于在这 个领域的杰出贡献, Heck, Negishi 和 Suzuki 三位化学家 在 2010 年被授予诺贝尔化学奖.

随着偶联反应不断的成熟和完善, 越来越多的化学 工作者开始意识到: 经典偶联反应中所使用的亲电试剂
多数都是由亲核试剂经过多步功能团化后转化而来, 这 在很大程度上制约了经典偶联反应的发展及其应用. 在 此背景下，直接使用亲核试剂构建各类化学键的氧化偶 联反应便应运而生 ${ }^{[3]}$. 与经典偶联反应相比, 氧化偶联 反应不仅减少了繁咒的底物前功能团化操作, 提高了反 应效率，而且其原料来源也更为广泛，大大增加了反应 的实用性，降低了生产成本. 值得一提的是，第三代氧 化偶联反应使用碳氢化合物作为亲核试剂，偶联反应之 后丢掉的只是两个氢原子, 从反应形式上看, 大大提高 了物质构建过程中的原子经济性, 更符合当今绿色化学 发展的宗旨. 目前, 氧化偶联反应的发展虽然还处于一 个初级阶段，但是其发展迅速，每年发表的《科学引文 索引》 $(\mathrm{SCI})$ 论文数量持续攀升, 表明氧化偶联反应已是

\footnotetext{
*E-mail: aiwenlei@whu.edu.cn

Received November 14, 2014; revised January 24, 2015; published online January 28, 2015.

Project supported by the 973 Program (Nos. 2012CB725302, 2011CB808600), the National Natural Science Foundation of China (Nos. 21390400, 21272180, and 21302148), and the Research Fund for the Doctoral Program of Higher Education of China (No. 20120141130002), the Program for Changjiang Scholars and Innovative Research Team in University (No. IRT1030), the Ministry of Science and Technology of China (No. 2012YQ120060) and the Program of Introducing Talents of Discipline to Universities of China (111 Program).

科技部 973 计划(Nos. 2012CB725302, 2011CB808600)、国家自然科学基金(Nos. 21390400, 21272180, 21302148)、高等学校博士学科点专项科研基金 资助(No. 20120141130002)、教育部长江学者和创新团队发展计划(No. IRT1030)、国家科学技术部基金(No. 2012YQ120060)、高等学校学科创新引智 计划(111 项目)资助项目.
} 
有机化学的热点研究领域之一.

主要综述了我们研究小组近几年来在氧化偶联领 域所取得的一些研究成果, 将分为三个部分进行论述: 氧化偶联反应进展、第三代氧化偶联反应、结论与展望; 其中着重介绍第三代氧化偶联反应.

\section{1 氧化偶联反应进展}

负负相斥是自然界的基本规律, 要实现两个亲核试 剂之间的偶联反应, 就需要往反应体系里面引入氧化 剂, 转移成键过程中多余的两个负电荷, 这从反应历程 上决定了其与经典偶联反应的本质不同. 近年来, 在金 属有机化学研究的推动下, 氧化偶联反应取得了许多突 破. 本部分将根据亲核试剂的不同来论述氧化偶联反应 的发展.

\section{1 第一代氧化偶联反应: 两个金属试剂作为亲核试 剂}

1993 年, Lipshutz 等 ${ }^{[4]}$ 首次实现了两个芳基金属试 剂的交叉偶联反应, 该反应中使用化学计量的 $\mathrm{CuCN}$ 和 氧气作为氧化剂在低温下实现了多种不对称联芳烃的 合成. 我们课题组 ${ }^{[5]}$ 于 2006 年报道了第一例烷基锌试剂 与炔基锡试剂的钯催化氧化偶联反应. 使用廉价的 2氯-2-苯基苯乙酮(desyl chloride)作为氧化剂在 $\operatorname{Pd}(\mathrm{dba})_{2}$ 催化下高选择性地得到了氧化交叉偶联产物(Eq. 1), 该 反应的底物适用范围也比较广泛, 各种脂肪链的烷基锌 试剂都可以取得中等到优秀的收率, 而且该反应体系还 可以兼容羰基化合物.

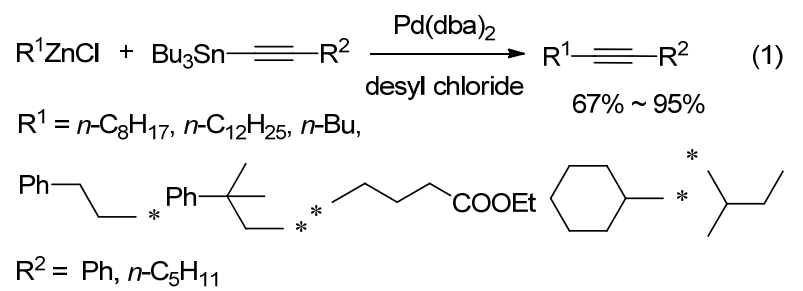

与经典偶联反应的机理不同 ${ }^{[3 \mathrm{a}, 3 \mathrm{c}]}$, 该反应中零价钯 首先被 2-氯-2-苯基苯乙酮(desyl chloride)氧化到二价钯 物种启动催化循环, 二价钯物种 $\mathbf{C}$ 分别经过与烷基锌试 剂和炔基锡试剂的两次转金属化后得到中间体 $\mathbf{E}$, 之后 中间体 $\mathbf{E}$ 经过还原消除即得到目标产物, 同时释放出零 价钯实现催化循环(Scheme 1). 我们的相关机理研究表 明, 该反应之所以高选择性地得到氧化交叉偶联产物, 其关键在于该反应条件下烷基金属试剂的自偶联反应 速度较为缓慢而炔基锡试剂的自偶联反应并不发生. 在 线红外跟踪反应显示烷基锌试剂选择性地与钯氧键发 生转金属化而炔基锡试剂则选择性地与钯氯键发生转 金属化. 值得指出的是, 该反应可以很好地兼容各种含

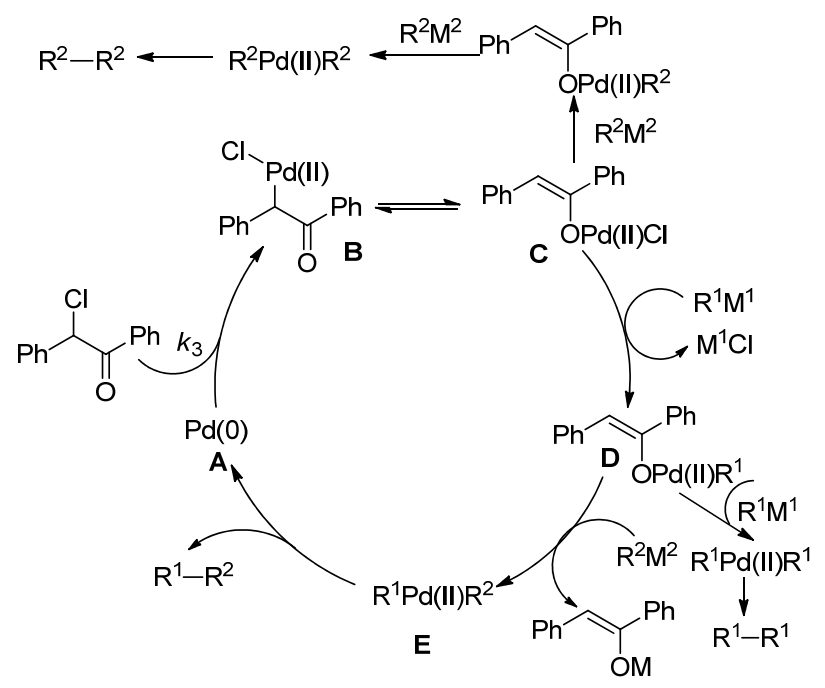

图式 1 烷基锌试剂与炔基锡试剂的氧化偶联反应可能的反 应机理

Scheme 1 Proposed mechanism for the oxidative cross-coupling of alkylzinc with alkynylstannanes

有 $\beta$-氢的烷基锌试剂, 其原因可能是联苯乙烯酮(DBA) 作为 $\pi$ 酸配体加速了反应的还原消除步骤，从而抑制了 $\beta$-氢消除步骤.

基于对该反应机理的深刻理解以及动力学行为的 认识和探究, 随后我们还高选择性地实现了芳基锌试剂 与烷基铟试剂的氧化交叉偶联反应(Eq. 2) ${ }^{[6]}$. 该反应对 于不同的一级以及二级铟试剂都可以取得很好的结果， 进一步丰富和拓展了氧化偶联反应的成键类型以及底 物适用范围，其反应机理与上面类似，不再赘述.

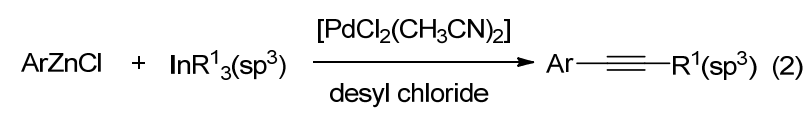

\section{2 第二代氧化偶联反应：碳氢化合物与金属试剂作 为亲核试剂}

第一代氧化偶联反应使用不同金属试剂作为亲核 试剂高选择性地得到氧化交叉偶联产物, 该方法为各种 化学键的构筑提供了一种新的成键模式. 然而从其原料 的来源和制备角度考虑，该类反应不够绿色经济，底物 范围也局限很多. 相反, 作为自然界普遍存在的碳氢化 合物不仅来源十分广泛，相对也更廉价易得，作为亲核 试剂实现氧化偶联反应，从反应形式上而言，最终丢掉 的原子更少，原子经济性也更高，实际应用也会更加广 泛. 例如 $\mathrm{Oi}$ 等 $^{[7]}$ 于 1998 年首次报道了芳烃和芳基金属 试剂的氧化偶联反应，反应中通过吡啶作为导向基团选 择性地在 2-芳基吡啶的芳基邻位 $\mathrm{C}-\mathrm{H}$ 键上与芳基锡试 剂发生作用实现芳基化. 基于这些考虑，在第一代氧化 偶联反应的基础上, 我们课题组发展了第二代氧化偶联 反应：即使用碳氢化合物与金属试剂作为亲核试剂实现 
氧化偶联反应.

2008 年, 我们课题组 ${ }^{[8]}$ 率先报道了使用烷基/芳基 取代的铟试剂与醇类的氧化羰基化反应(Eqs. 3, 4), 仍 然使用了廉价的 2-氯-2-苯基苯乙酮(desyl chloride)作为 氧化剂, 在 $60{ }^{\circ} \mathrm{C}$ 和 $0.34 \mathrm{MPa}$ 一氧化碳压力条件下即可 顺利地实现氧化羰基化反应且不需要额外的碱, 对于 醇、伯胺等亲核试剂都可以取得满意的结果. 该反应不 仅弥补了烷基卤代物由于对低价金属氧化加成困难而 不易实现羰基化反应的缺陷 ${ }^{[9]}$, 而且对于二级芳基铟试 剂的反应也可顺利进行. 同时, 该反应很好地抑制了 $\beta$ 氢消除步骤 $[9 \mathrm{a}, 10]$, 对于一级以及二级铟试剂都可以顺利 参与反应. 由于铟试剂本身较弱的碱性以及温和的反应 活性，该反应底物的官能团兼容性效果也非常好.

$$
\begin{aligned}
& \mathrm{R}_{n}{ }^{1} \operatorname{InX} X_{3-n}+\mathrm{R}^{2} \mathrm{OH}+\mathrm{CO} \underset{\mathrm{Pd} / \text { ligand }}{\text { desyl chloride }} \mathrm{R}^{1}-\mathrm{COOR}^{2} \\
& \mathrm{R}^{1}=\text { alkyl, aryl; } n=1,2,3 \\
& \mathrm{R}^{2}=\mathrm{Me}, \mathrm{Et}, n-\mathrm{Bu} \\
&
\end{aligned}
$$

随后, 我们运用在线红外等手段对该反应的机理进 行了研究, 认为该反应首先由 2-氯-2-苯基苯乙酮(desyl chloride) 将零价钯氧化到二价钯启动催化循环, 经过醇 解以及一氧化碳插入步骤得到中间体 $\mathbf{C}$, 再经过转金 属化和还原消除步骤即得到产物并释放出零价钯实现 催化循环(Scheme 2).

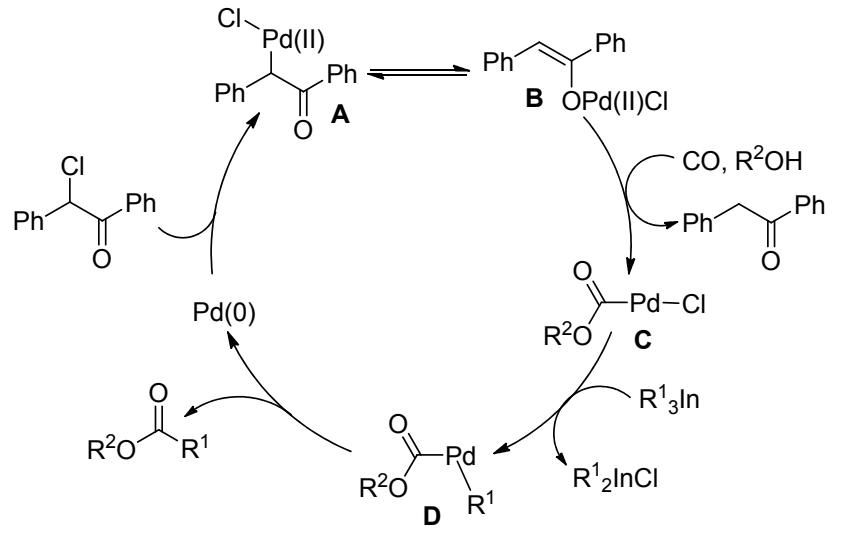

图式 2 烷基/芳基取代的铟试剂的氧化羰基化反应的推测机 理

Scheme 2 Speculated reaction pathways of the oxidative carbonylation of aryl and alkyl indium reagents

在此基础上, 2010 年, 我们 ${ }^{[11]}$ 又首次实现了芳基硼 酸酯的氧化羰基化反应(Eq. 5). 该反应在 $40 \sim 50{ }^{\circ} \mathrm{C}$ 即 可顺利进行, 使用一个大气压的一氧化碳以及使用氧气
作为氧化剂，通过调控二者的比例可以很好地控制羰基 化反应的选择性，大大提高了反应的实用性.

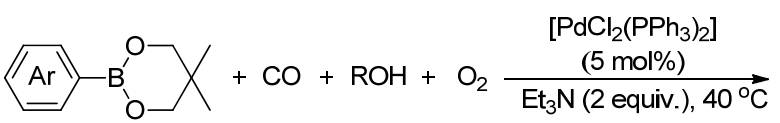

$\mathrm{R}: \mathrm{Me}, \mathrm{Et}, i-\mathrm{Pr}, n-\mathrm{Bu}$, etc.<smiles>[R]OC(=O)c1ccccc1</smiles>

我们的研究结果表明, $\mathrm{PdCl}_{2}\left(\mathrm{PPh}_{3}\right)_{2}$ 并不能与芳基 硼酸酯直接进行转金属化, 其作为催化剂的前体需要先 转化为催化活性物种 $\left[\left(\eta-\mathrm{O}_{2}\right) \mathrm{Pd}\left(\mathrm{PPh}_{3}\right)_{2}\right]$ 才能启动催化循 环，实现与芳基硼酸酯的转金属化. 同时，我们推测一 氧化碳在体系中不仅充当反应底物的角色, 而且作为 $\pi$ 酸配体很好地抑制了芳基嗍酸酯的氧化自偶联反应 ${ }^{[12]}$. 与传统的使用卤代物实现羰基化反应相比 ${ }^{[13]}$, 使用氧 化偶联方法避免了一氧化碳作为 $\pi$ 酸配体抑制卤代物对 低价金属氧化加成步骤 ${ }^{[14]}$, 从而使反应条件更加温和, 实用性更强.

由于烷基卤代物与低价金属的氧化加成相对比较 困难且很容易发生 $\beta$-氢消除而产生副产物, 迄今为止, 烷基碳链的偶联反应一直是有机合成化学的一大挑 战 ${ }^{[15]}$, 该类反应中往往需要加入过量的重金属或有机 氧化剂，反应条件苛刻. 我们课题组使用不同的烷基金 属试剂实现了多种氧化交叉偶联反应, 很好地弥补了烷 基卤代物在偶联反应方面上的不足. 2010 年，我们课题 组 ${ }^{[16]}$ 高选择性地实现了炔烃化合物与烷基锌试剂的氧 化交叉偶联反应(Eq. 6), 该反应直接使用空气作为氧化 剂, 对于各种一级与二级锌试剂都能得到很好的结果. 反应体系中的一氧化碳作为 $\pi$ 酸配体加速了炔烃化合物 与烷基锌试剂的氧化交叉偶联的还原消除步骤，对反应 的高选择性起到了至关重要的作用 ${ }^{[17]}$.

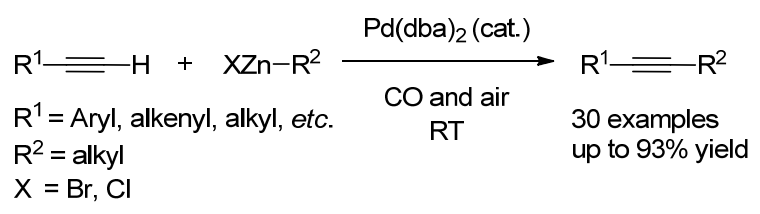

在线红外动力学检测表明, 反应过程中氧化交叉偶 联的反应速度要远远快于炔烃的氧化自偶联速度是其 高选择性的原因之一。同时，我们还检测到低浓度的炔 基锌试剂在反应过程中会缓慢产生，之后又逐渐消失， 这说明在该反应条件下只有低浓度的炔基锌试剂缓慢 产生是炔烃氧化自偶联速度相对很慢的主导因素, 这也 直观地揭示了该反应高选择性的原因所在. 


\section{2 第三代氧化偶联反应：碳氢物种作为亲核试 剂}

不论是从原料的来源还是原子利用率角度考虑, 从 反应形式上, 直接使用两个碳氢化合物实现氧化交叉偶 联反应无疑是更为理想的构建物质的方式 ${ }^{[3]}$. 在该研究 领域内, 已有许多杰出的成果. 如在 1993 年 Murata 等 ${ }^{[18]}$ 通过 $\mathrm{Cu} / \mathrm{O}_{2}$ 条件首次实现了烷烃和炔烃的氧化偶联 反应, Fuchita 等 ${ }^{[19]}$ 于 2001 年在研究芳基金化合物地过 程中也首次实现了芳烃和炔烃之间的氧化偶联反应, 此 外在 2004 年 Sanford 等 ${ }^{[20]}$ 还在钯催化条件下实现了芳烃 和烷烃与醇或羧酸的高选择性 $\mathrm{C}-\mathrm{O}$ 成键反应. 通过对 氧化偶联反应的进一步深入思考, 我们课题组发展了第 三代氧化偶联反应，即直接使用不同的碳氢化合物实现 氧化交叉偶联反应, 减少原子浪费的同时, 使反应更加 高效, 进一步弥补前两代氧化偶联反应的不足. 本部分 将根据反应类型的不同来论述第三代氧化偶联反应, 主 要包括: 炔烃参与的氧化偶联反应、氧化羰基化反应、 氧化酯化反应、碳杂成键的氧化偶联反应四个部分.

\section{1 炔烃参与的氧化偶联反应}

炔烃是自然界中广泛存在和廉价易得的化合物, 也 是有机合成中重要的中间体, 基于炔烃的功能团化反应 已经成为有机合成中的重要方法 ${ }^{[21]}$. 目前为止, 大多数 的炔烃的功能团化反应主要集中在内炔上, 这是由于端 炔化合物在过渡金属催化条件下反应活性较高, 极易发 生自身偶联反应, 从而导致反应选择性差、效率低等非 预期的不利结果 ${ }^{[22]}$. 考虑到端炔化合物的重要应用价 值以及对端炔化合物浓厚的研究兴趣, 我们在端炔参与 的氧化偶联反应方面取得了一些研究成果, 介绍如下.

作为天然产物、医药中间体以及电子发光体的重要 骨架结构的共轭二炔化合物, 其制备一直以来都是化学 合成领域的研究热点 ${ }^{[23]}$. 尽管 Glaser 早在 1869 年就报 道了炔烃化合物的自偶联反应 ${ }^{[21 a, 21 b]}$, 但是对于不对称 共轭二炔化合物的高效合成则是直到 20 世纪 70 年代才 发展起来的 Cadiot-Chodkiewicz 反应 ${ }^{[24]}$, 该反应使用炔 卤化合物与炔烃在铜催化条件下实现了交叉偶联反应. 由于其中作为反应底物的炔卤化合物是由炔烃经过卤 化制备而来, 因此, 不论是从经济角度还是环境友好方 面考虑，直接使用不同炔烃化合物通过氧化交叉偶联反 应合成非对称共轭二炔化合物将是更为理想的方式.

2008 年, 我们课题组 ${ }^{[25]}$ 实现了不同炔烃的氧化交 叉偶联反应 (Eq. 7), 该反应使用廉价的镍盐和铜盐共 同作为催化剂, 利用氧气/空气作为氧化剂在室温条件 下即高效地实现不对称共轭二炔化合物的合成. 基于我 们对共轭二炔化合物的浓厚兴趣, 在反应之初, 我们首
先探究了单独使用碘化亚铜作为催化剂催化苯乙炔的 自偶联反应, 结果反应一个小时我们以 $25 \%$ 的收率得到 了预期的苯乙炔自偶联产物，而在加入镍催化剂之后， 同样的条件下反应的收率则大幅度地提高到了 $93 \%$, 该 实验说明铜盐和镍盐在反应过程中可能起到了协同作 用，二者共催化可以大幅度提高反应的速率. 基于这个 发现，我们开始以苯乙炔和乙酸丙炔酯的交叉偶联反应 作为模板进行了系统的条件笁选. 实验研究表明, 炔烃 的数学统计分布和镍催化剂的引入对于高效实现氧化 交叉偶联反应起到了决定作用，在苯乙炔/乙酸丙炔酯 的比例较低以及不加镍催化剂的条件下得到交叉偶联 的收率会很低，最终我们在苯乙炔 5 倍量的情况下高选 择性地得到了交叉偶联产物. 该反应在底物的普适性方 面也取得了良好的结果，对于在过渡金属催化反应中往 往很难兼容的芳基碘代物以及溴代物都可以取得很好 的结果.

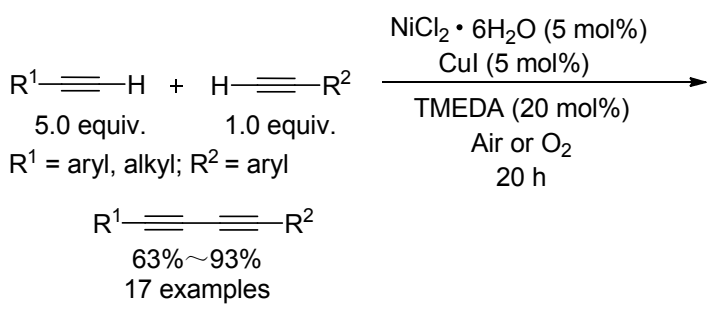

基于一价铜与炔烃作用相比零价镍与炔烃作用更 加容易的文献报道 ${ }^{[26]}$, 我们推测该反应首先由镍催化 剂与氧气作用得到镍氧中间体 $\mathbf{B}^{[27]}$, 经过与在线生成的 炔铜物种 $\mathbf{C}$ 的两次转金属化后得到中间物种 $\mathbf{D}$, 中间物 种 D 再经过还原消除即得到目标产物，同时释放出镍催 化剂 $\mathbf{A}$ 实现催化循环(Scheme 3) ${ }^{[28]}$.

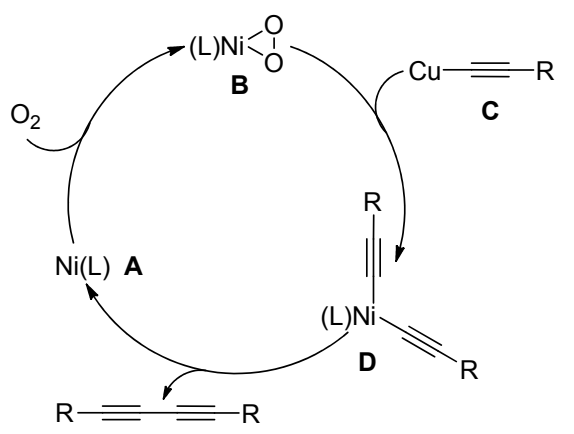

图式 3 不同炔烃的氧化交叉偶联反应的推测机理 Scheme 3 Speculated reaction pathways of alkyne couplings

为了证明镍催化剂的作用以及炔基金属物种的产 生, 我们使用炔基锌试剂(Eq. 8)或者通过加入氯化锌原 位生成炔基锌试剂(Eq. 9)进行反应，最终取得了良好的 结果. 


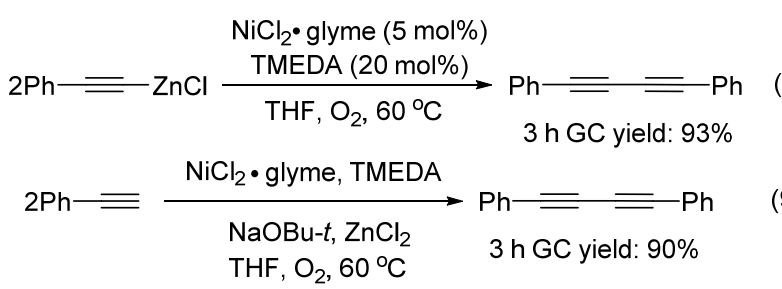

文献报道的关于铜催化的炔烃的自偶联反应中, 得 到一分子的产物需要消耗两分子的氧气 ${ }^{[29]}$. 而我们提 出的机理中, 得到一分子的产物只需要消耗一分子的氧 气. 为了进一步理解该氧化交叉偶联反应的反应历程, 我们使用 $100 \mathrm{mmol}$ 的苯乙炔做了氧气与产物之间的动 力学实验. 其结果证明: 在该反应条件下, 氧气的消耗 和产物的生成是单倍的关系, 而非倍数关系, 佐证了我 们机理的合理性(图 1).

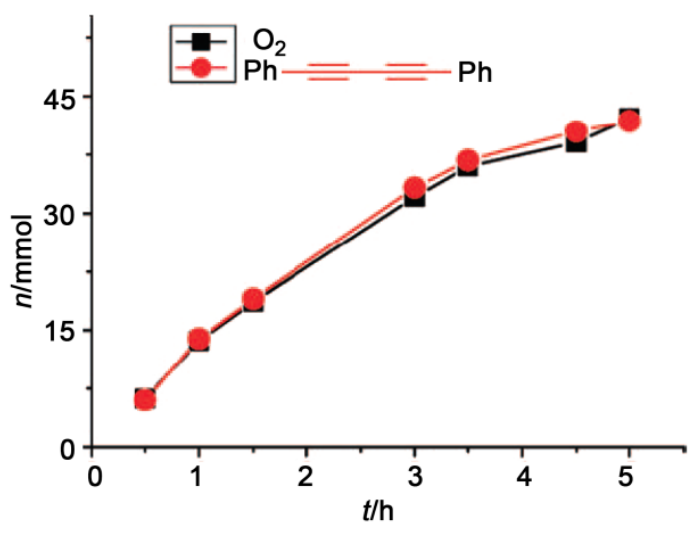

图 $1 \mathrm{Ni}(\mathrm{II}) / \mathrm{Cu}(\mathrm{I})$ 催化的苯乙炔 $(100 \mathrm{mmol})$ 在四氢呋喃溶液中 的自偶联反应的时况图

Figure 1 Time profiles of homocoupling of benzylacetylene (100 mmol) catalyzed by $\mathrm{Ni}(\mathrm{II}) / \mathrm{Cu}(\mathrm{I})$ in THF in $500 \mathrm{~mL}$ Schlenk flask

Red line represents the amount of diphenylacetylene versus time; black line represents the consumed $\mathrm{O}_{2}$ versus time

在我们成功地实现了 $\mathrm{C}_{\mathrm{sp}}-\mathrm{C}_{\mathrm{sp}}$ 的氧化交叉偶联反应 之后, 是否可以直接使用炔烃化合物与羰基化合物实现 $\mathrm{C}_{\mathrm{sp}}-\mathrm{C}_{\mathrm{sp}} 3$ 的氧化交叉偶联反应引起了我们的关注. 基于 这样的实验设想, 我们使用苯乙炔和乙酰丙酮在铜盐作 氧化剂的条件下尝试了 $\mathrm{C}_{\mathrm{sp}}-\mathrm{C}_{\mathrm{sp}} 3$ 的氧化交叉偶联反应, 但是在大多数情况下的主要产物都是苯乙炔的自偶联 产物. 令人惊喜的是, 当使用银盐代替铜盐作为氧化剂 时，我们以较好的收率高选择性地得到了多取代的呋喃 结构产物，而且没有观察到炔烃氧化自偶联产物的生成 (Scheme 4) ${ }^{[30]}$. 我们推测可能是银盐的存在使后续的环 异构化反应更容易进行 ${ }^{[31]}$, 进而得到了氧化交叉偶联环异构化串联反应的产物。

多取代呋喃以及吡咯衍生物不仅是天然产物、医 药、农药等化合物的重要骨架结构, 也是有机合成化学

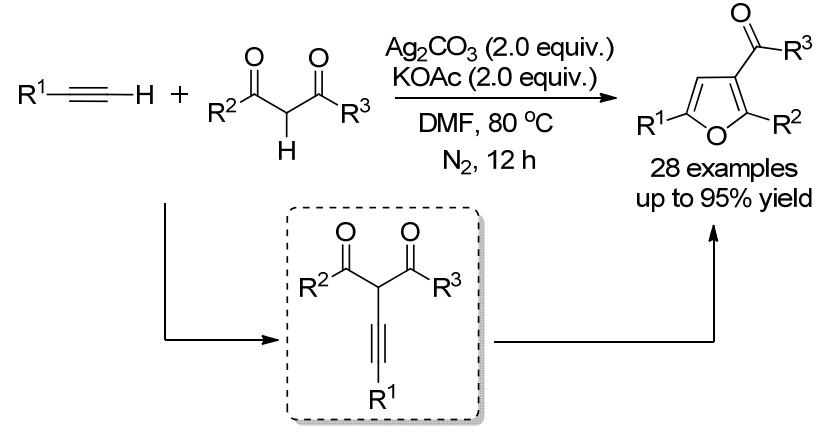

图式 4 银促进的端炔与 1,3-二羰基化合物氧化交叉偶联反应 及其可能的反应途径

Scheme 4 Silver-mediated oxidative cross-coupling reaction of terminal alkynes with 1,3-dicarbonyl compounds and its possible reaction pathways

中重要的中间体，如何从简单原料出发实现区域选择性 地合成多取代呋喃以及吡咯衍生物一直都是合成化学 家们关注的焦点 ${ }^{[32]}$. 我们直接使用简单易得的碳氢化 合物通过一锅法反应高选择性地实现多取代呋喃的合 成无疑是十分吸引人的，该反应适用于各种芳香族取代 的端炔以及各类 1,3-二羰基化合物，对于个别的脂肪族 炔烃也能得到相应的产物. 值得一提的是, 利用该方法 还可以合成多取代的吡咯化合物(Eq. 10).

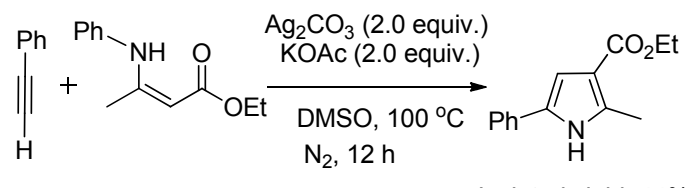

反应过程使用的化学计量的碳酸银在反应结束后 通过简单的过滤、硝酸酸化以及碳酸钠的处理可以重复 回收使用且催化活性并没有发生变化(Scheme 5) ${ }^{[33]}$, 这 也在一定程度上增加了该反应的实用性.

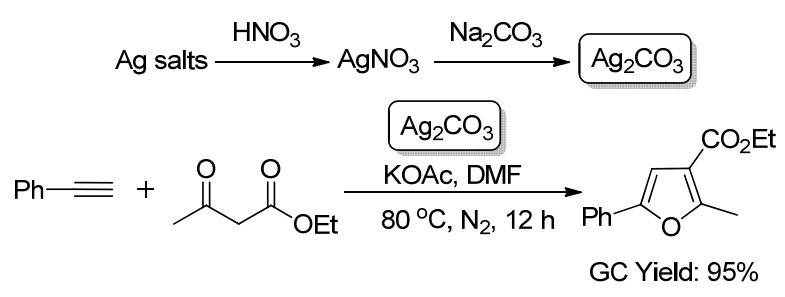

图式 5 银盐的回收实验

Scheme 5 Recovery experiment of Ag salts

我们对该反应的可能的机理进行了初步的探索. 根 据端炔与银盐作用可能会生成炔银物种的相关文献报 道 ${ }^{[34]}$, 我们推测在该反应体系中, 炔银物种可能是反应 的重要中间体. 因此我们使用提前制备好的炔银在没加 碳酸银的条件下与乙酰乙酸乙酯反应，结果只得到了很 
少量的呋喃产物. 而在该条件下额外加入 2 倍量的碳酸 银则能以中等收率得到目标产物(Eq. 11), 这表明炔银 可能是反应的中间体. 同时在标准条件下使用苯乙烯代 替苯乙炔参与反应, 发现并没有得到二氢呋喃产物, 因 此也排出了氧化自由基途径的可能性 ${ }^{[35]}$. 目前, 该反应 的机理仍在研究之中.

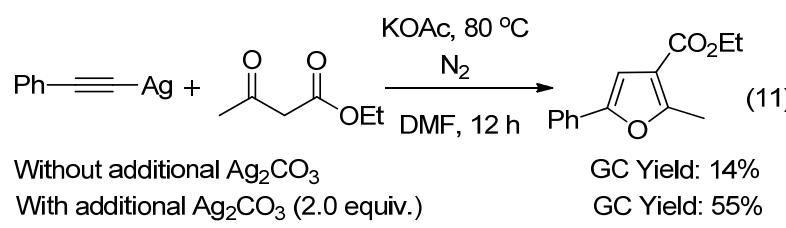

咪唑杂环类化合物是天然产物以及生物活性分子 中的一类重要骨架结构 ${ }^{[36]}$, 其中具有药理学性质的咪 唑并 $[1,2-\alpha]$ 吡啶类化合物是一些广为使用的重要药物如 唑吡坦、阿吡坦、佐利米定的有效成分中的关键骨架部 分 ${ }^{[37]}$, 尽管它们的合成方法已经有所报道, 但是直接使 用简单易得的碳氢化合物如吡啶衍生物和炔烃作为原 料无疑是十分吸引人的 ${ }^{[36 \mathrm{a}, 38]}$. 在实现 $\mathrm{C}-\mathrm{H} / \mathrm{C}-\mathrm{H}$ 氧化 交叉偶联/环化反应的启示下, 我们推测 $\mathrm{C}-\mathrm{H} / \mathrm{N}-\mathrm{H}$ 氧 化交叉偶联/环化反应也是很有可能进行的, 即使用 2氨基吡啶与苯乙炔反应合成咪唑杂环类化合物是可行 的. 正如我们预期的一样, 经过系统的条件优化过程, 2-氨基吡啶与苯乙炔在银盐的促进下很好地得到了咪 唑并 $[1,2-\alpha]$ 吡啶 (Eq. 12) ${ }^{[39]}$. 该反应使用银盐作为促进 剂很好地避免了苯乙炔的氧化自偶联产物以及其他副 产物的产生, 底物的普适性也较好, 各类取代的 2-氨基 吡啶衍生物以及芳香炔类都可顺利得到产物, 个别的脂 肪族炔烃也能参与反应. 官能团兼容性方面也很好, 可 以兼容芳基氯代物、溴代物、酯基以及噻吩等. 值得一 提的是由于反应活性较高, 在过渡金属催化体系中常常 不能兼容的芳基碘代物在该条件下也能很好兼容, 为该 类化合物的后续官能团化提供了可能. 反应过程中使用 的化学计量的碳酸银在反应结束之后经过简单的后处 理仍然可以循环使用 ${ }^{[40]}$.

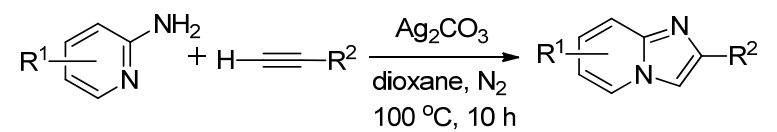

利用该方法，我们使用对甲硫醚取代的苯乙炔与 2氨基吡啶反应以中等收率得到了相应的咪唑类化合物 3ak, 化合物 3ak 再经过简单的氧化即可以中等的收率 得到药物分子佐利米定(Scheme 6).

我们对反应的机理也进行了初步的探究, 当使用诸 如二苯乙炔、1-苯基-1-丙炔等内炔作为底物在标准条件 下并没有得到目标产物. 而直接使用提前制备好的炔银

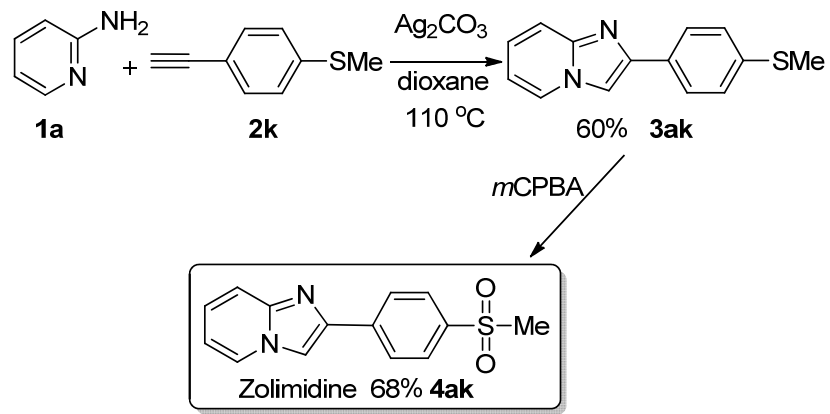

图式 6 佐利米定的合成 Scheme 6 Preparation of zolimidine

在不加碳酸银的条件下只得到很少产物, 额外加入 2 倍 量的碳酸银则能以中等收率得到目标产物(Eq. 13), 这 表明炔银可能是反应的中间体.

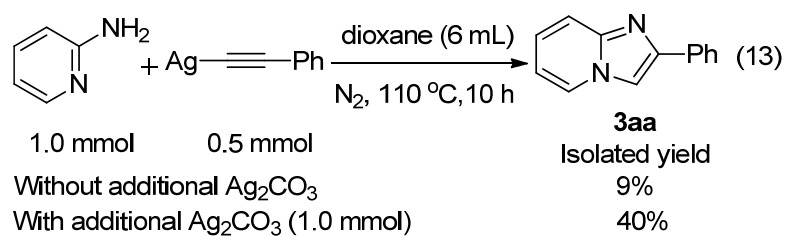

据此我们推测该反应可能先是炔与银作用生成炔 银中间体 $\mathbf{A}$, 之后按照其他相关文献中所描述的在银辅 助下 2-氨基吡啶对炔银 $\mathbf{A}$ 进行亲核进攻得到关键中间 体 $\mathbf{B}^{[41]}$ ，中间体 $\mathbf{B}$ 再经过银诱导的氧化环化反应即得到 最终产物 3aa (Scheme 7). 目前, 该反应的机理仍在进 一步的研究之中.

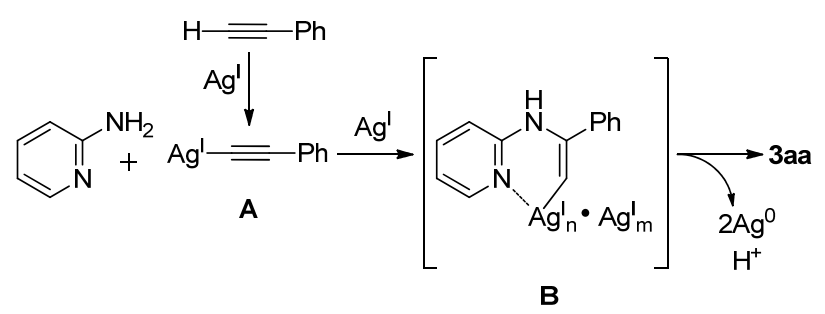

图式 7 银促进的 2-氨基吡啶与端炔氧化交叉偶联/环化反应 的可能机理

Scheme 7 Proposed mechanism of silver-mediated oxidative cross-coupling/cyclization between 2-aminopyridines and terminal alkynes

\section{2 氧化羰基化反应}

过渡金属催化的有机卤代物的羰基化反应是在 20 世纪 60 年代由 Heck 等发展起来的 ${ }^{[42]}$, 其在过去的五十 年间被广泛应用于工业以及有机合成之中，逐渐成为高 效高选择性的构建各种羰基化合物的基本方法 ${ }^{[43]}$. 然 而，一氧化碳作为 $\pi$ 酸配体会使低价金属如零价钯变得

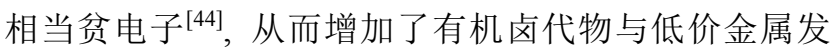
生氧化加成的难度. 因此, 该类羰基化反应往往需要在 
较高的温度以及较大的一氧化碳的压力等苛刻条件下 才能进行 ${ }^{[45]}$, 这在一定程度上限制了该类羰基化反应 的发展和应用. 此外, 有机卤代物一般都是由相应的碳 氢化合物制备而来, 这也使得整个化学合成步骤较为兄 长, 大大降低了反应的原子经济性. 相比之下, 直接使 用碳氢化合物实现羰基化反应不仅可以减少化学合成 步骤, 提高原子经济性, 使反应更为环境友好, 而且该 类氧化羰基化反应避免了高浓度一氧化碳条件下的困 难的有机卤代物与低价金属的氧化加成步骤, 可以使反 应在更温和的条件下顺利进行 ${ }^{[46]}$. 在我们实验室开发 的金属试剂作为亲核试剂的氧化羰基化反应的基础上 ${ }^{[8,11]}, 2011$ 年, 我们报道了杂环化合物作为亲核试剂的 氧化羰基化反应(Eqs. 14 16 $)^{[47]}$, 合成了普遍存在于药 物分子以及其他众多有用化学分子中的重要结构单 元一一杂环酯类化合物 ${ }^{[48]}$. 该反应使用氧气作为氧化 剂, 在常压一氧化碳压力下不需要加碱就可以高选择性 地在吲哚的 3-位或是噻吩的 2-位实现氧化羰基化反应. 此外, 未保护的吲哚的 $\mathrm{N}-\mathrm{H}$ 也可以很好地参与反应得 到氨基甲酸酯类化合物. 对于杂环底物, 不论是含有富 电子还是缺电子取代基都可以得到相应产物. 对于醇类 底物, 一级、二级醇以及芐醇类都是很好的反应底物.
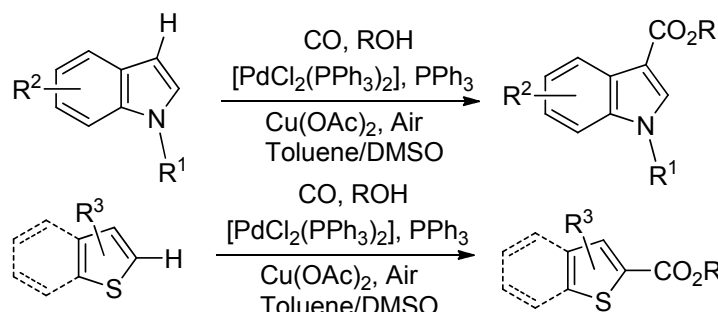<smiles>[R]OC(=O)c1cc2ccccc2s1</smiles>
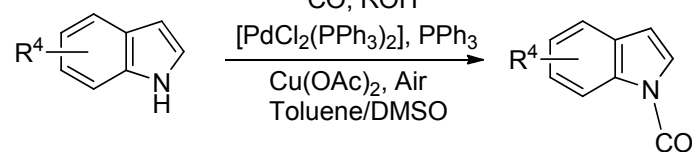

基于反应活性位点高选择性地发生在杂化化合物 的富电性位置的实验现象以及相关的文献报道 ${ }^{[49]}$, 我 们推测反应首先是杂环化合物与二价钯物种 $\mathbf{B}$ 经过亲 电钯化过程得到中间体 $\mathbf{C}$, 再经过一氧化碳插入以及醇 的转金属过程后, 经还原消除后得到目标产物并释放出 零价钯物种 $\mathbf{A}$, 零价钯在铜和氧气的作用下重新得到二 价钯物种实现催化循环 (Scheme 8).

在实现了 $\mathrm{C}_{\mathrm{sp}^{2}}-\mathrm{H} / \mathrm{O}-\mathrm{H}$ 氧化羰基化反应之后, $\mathrm{C}_{\mathrm{sp}}{ }^{2}-\mathrm{H} / \mathrm{C}_{\mathrm{sp}}{ }^{2}-\mathrm{H}$ 的氧化羰基化反应引起了我们浓厚的 研究兴趣, 相比之下, 后者的反应难度更大. 这是因为 芳环化合物的亲核性和醇类化合物相差很大, 在转金属 化过程中相对要困难很多. 迄今为止, 双碳氢键的羰基 化反应仍旧是该领域中所面临的巨大挑战之一 ${ }^{[50]}$. 基

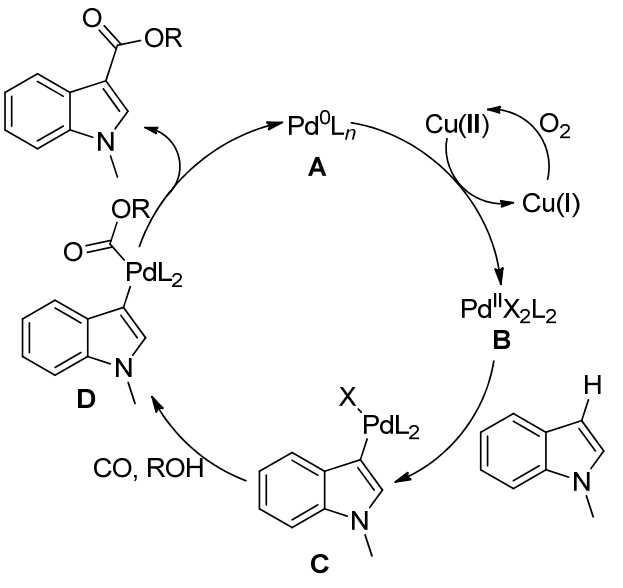

图式 8 杂环化合物的氧化羰基化反应的可能机理 Scheme 8 Proposed mechanism of oxidatve carbonylation of heteroarenes

于我们对氧化偶联反应 ${ }^{[49 c]}$ 以及羰基化反应 ${ }^{[51]}$ 上的深刻 认识, 我们将目光瞄准在二芳基醚类化合物的氧化羰基 化反应上. 这主要基于两方面的考虑: 其一, 二芳基醚 类化合物的两个芳环都属于相对富电性芳环，与贫电子 钯物种都可以较为容易地发生亲电钯化, 分子内实现氧 化羰基化反应的可能性很大; 其次, 我们预期得到的产 物是占吨酮类化合物, 它们广泛存在于自然界以及人工 合成的药物中, 具有很显著的生物和药物活性, 从化学 到生物乃至药学领域都具有很广泛的应用, 是一种非常 重要的结构单元 ${ }^{[52]}$. 传统的合成占吨酮的方法往往步 骤繁琐且条件苛刻 ${ }^{[53]}$, 相比之下, 我们设计的合成方法 优势则非常明显. 基于以上设想, 我们 ${ }^{[54]}$ 最终使用三氟 乙酸作为溶剂, 醋酸钯作为催化剂, 过硫酸钾作为氧化 剂, 在常压温和的条价下即以良好的收率得到了各种占 吨酮类化合物(Eq. 17).

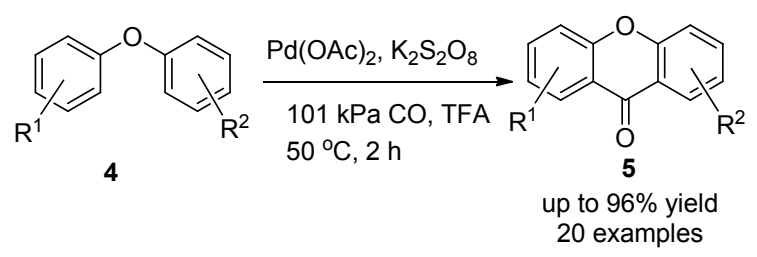

我们对该反应的机理也进行了探究，使用在线红外 检测发现不同的底物浓度与反应速率皆呈现零级的关 系(图 2), 即反应过程中反应速率不随反应底物浓度的 降低而变化. 这表明第一个碳氢键断裂步骤不是反应的 决速步. 另外, 我们使用对二甲苯基醚作为底物, 通过 在线红外检测发现: 一氧化碳的浓度对反应速率没有影 响(图 3). 从而排除了一氧化碳参与的步骤是反应的决 速步.

根据文献相关报道, 我们了解到 $\operatorname{ArCOPdAr}$ 物种的 还原消除步骤是个容易进行的过程 ${ }^{[5]}$, 因此推断: 第二 


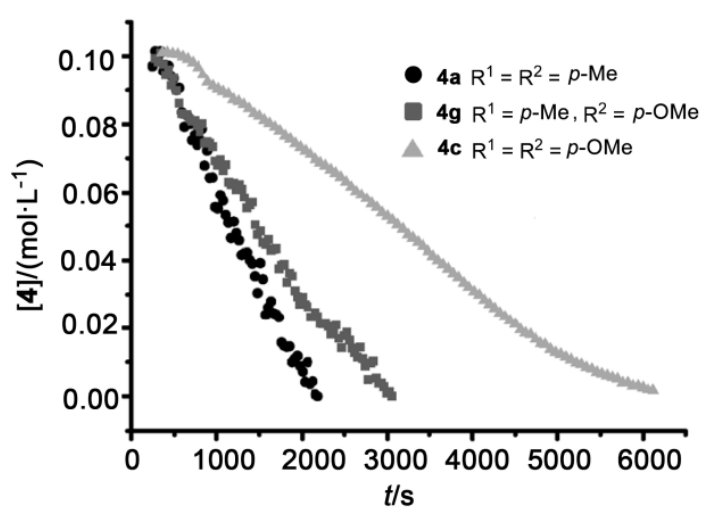

图 2 不同底物的双 $\mathrm{C}-\mathrm{H}$ 键氧化羰基反应化的动力学谱图

Figure 2 Kinetic plots of oxidative double $\mathrm{C}-\mathrm{H}$ carbonylation of different substrates

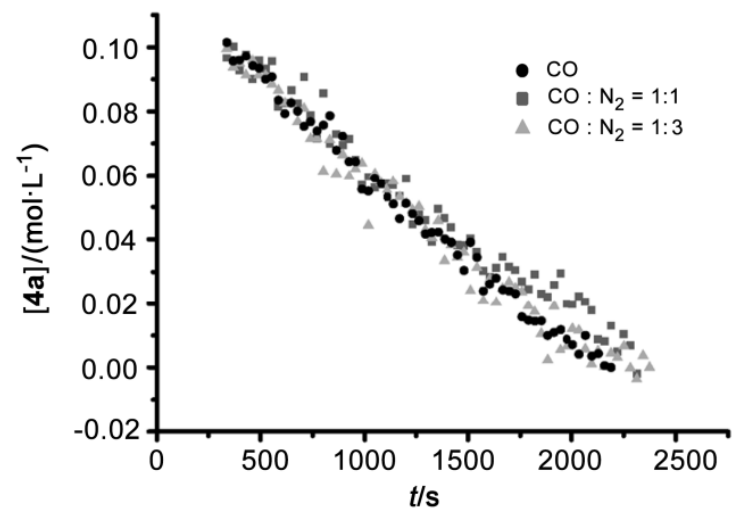

图 3 对二甲苯基醚在不同一氧化碳压力下的双 $\mathrm{C}-\mathrm{H}$ 键氧化 羰基化的动力学谱图

Figure 3 Kinetic profiles of oxidative double $\mathrm{C}-\mathrm{H}$ carbonylation of 4-tolyl ether with different $\mathrm{CO}$ pressures

个碳氢键断裂步骤可能是反应的决速步. 根据以上信息 以及之前的相关研究报道 ${ }^{[56]}$, 我们认为该反应的机理 可能为: 三氟乙酸与醋酸钯发生阴离子交换从而在线生 成贫电子的三氟醋酸钯 $\mathbf{A}$, 之后三氟醋酸钯 $\mathbf{A}$ 和二芳基 醚通过快速的亲电钯化过程得到芳基钯物种 $\mathbf{B}$, 接着一 氧化碳插入得到中间体 $\mathbf{C}$, 中间体 $\mathbf{C}$ 进一步通过分子内 碳氢键功能化得到中间体 $\mathbf{D}$, 随后中间体 $\mathbf{D}$ 经过还原消 除即得到目标产物并释放出零价钯, 产生的零价钯经过 硫酸钾氧化后重新得到三氟醋酸钯实现催化循环 (Scheme 9).

\section{3 氧化酯化反应}

酯基是天然产物、聚合物、各种化学药品中极其重 要的官能团结构 ${ }^{[57]}$, 其传统的合成方法主要包括卤代 物的羰基化反应 ${ }^{[58]}$ 、羧酸衍生物与醇的反应 ${ }^{\left[{ }^{[9]}\right.}$ 以及醛与 醇的氧化酯化反应 ${ }^{[00]}$ 等. 这些方法往往都需要经过多 步反应制备最初的原料, 反应过程中常常伴有不需要的

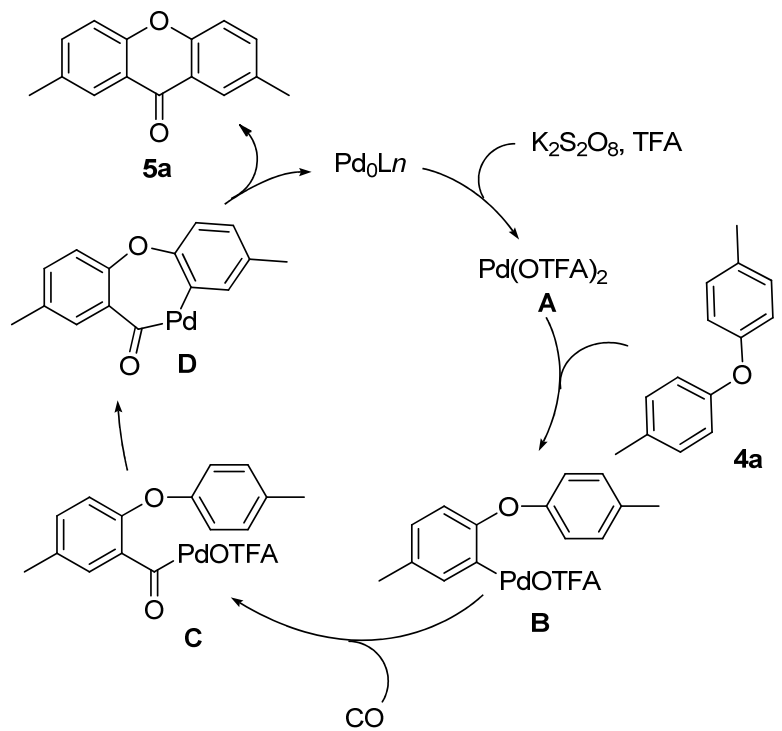

图式 9 二芳基醚类化合物的双 $\mathrm{C}-\mathrm{H}$ 键氧化羰基化反应的可 能机理

Scheme 9 Proposed reaction mechanism of oxidative double $\mathrm{C}-\mathrm{H}$ carbonylation of diaryl ethers

副产物, 有时还需要高温强氧化剂等苛刻的反应条件. 因此，如何发展绿色的符合可持续发展观的酯类化合物 的合成方法来替代传统合成方式是化学界的一大挑战. 作为广泛存在且廉价易得的醇类化合物, 如果可以绕过 传统的多步合成过程, 以直接转化方式合成酯类化合 物, 将会是一个上佳的选择 ${ }^{[61]}$. 正式基于这样的设计理 念, 2011 年, 我们 ${ }^{[2]}$ 首次实现了钯催化的醇类化合物的 有氧氧化交叉成酯反应(Eq. 18). 该反应使用氧气作为 氧化剂, 在温和的条件下以可以合成各类芳香甲酸酯类 化合物.

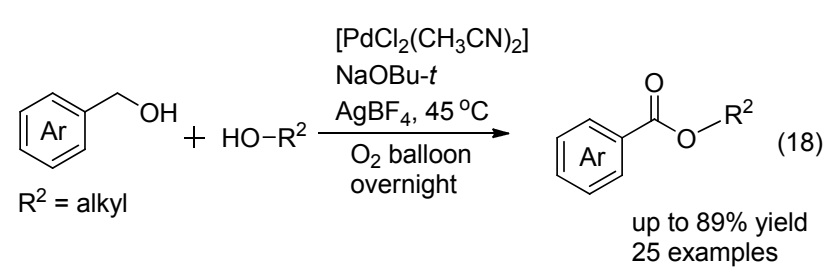

反应之初，我们使用茮醇与甲醇作为模板反应来进 行条件优化过程, 最终使用 $\mathrm{PdCl}_{2}\left(\mathrm{CH}_{3} \mathrm{CN}\right)_{2}$ 作为催化剂 前体，叔丁醇钠作为碱，在氧气作为氧化剂的条件下以 $74 \%$ 的收率得到目标产物. 之后, 我们在此最优条件下 进行底物扩展的时候发现: 芐醇苯环的电子效应对反应 影响很大，富电子的对甲氧基茮醇作为底物时其转化率 很高, 但是主要产物为对甲氧基苯甲醛 $8 \mathrm{~b}$, 而贫电子的 对硝基苄醇 $(\mathbf{6 c})$ 参与反应时没有检测到相应的对硝基苯 甲醛 $(\mathbf{8 c})$ 的生成, 但其转化率很低. 使用相应的苯甲醛 类作为反应底物对照发现: 在相同的条件下，富电子的 对甲氧基苯甲醛 $(\mathbf{8 b})$ 作为反应底物时的转化率很低, 对 
甲氧基苯甲醛多数剩余没有转化为相应的酯 $\mathbf{7 b}$, 而贫 电子的对硝基苯甲醛作为底物时则可以很好的转化为 相应的酯 7c (Scheme 10).

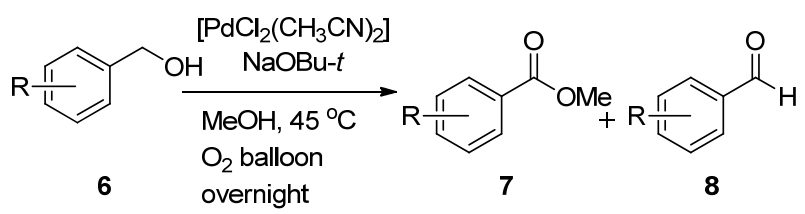

a: $\mathrm{R}=\mathrm{H}, 62 \% \mathbf{7 a}$; no $8 \mathbf{a}$

b: $\mathrm{R}=\mathrm{OMe}, 20 \% \mathbf{7 b} ; \mathbf{7 6} \% \mathbf{8 b}$

c: $\mathrm{R}=\mathrm{NO}_{2}, 54 \%$ 6c (unreacted); $36 \% 7 \mathrm{c}$

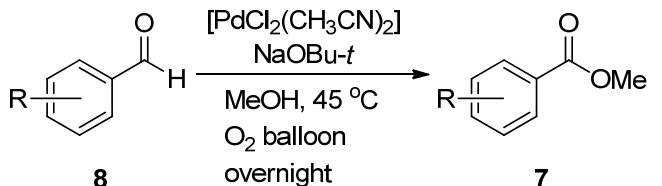

a: $\mathrm{R}=\mathrm{H}, 75 \% \mathbf{7 a}$

b: $R=O M e, 14 \% 7 b ; 68 \% 8 b$ (unreacted)

c: $\mathrm{R}=\mathrm{NO}_{2}, 88 \% 7 \mathrm{c}$

图式 10 氧化酯化反应的取代基效应

Scheme 10 Substitution effect on the oxidative esterification reaction

根据这样的结果我们推断, 醇的氧化交叉成酯的反 应历程可能包括两个过程. 芰醇类化合物在第一步中与 二价钯配位经过 $\beta$-氢消除后得到相应的苯甲醛类化合 物; 第二步则是原位生成的芳香醛类化合物与甲醇反应 得到半缩醛类化合物, 该半缩醛与钯配位经过 $\beta$-氢消除 后即得到最终的酯(Scheme 11). 在第一个过程中, 对于 富电子的茮醇更容易发生 $\beta$-氢消除得到相应的醛, 而对 于贫电子的贲醇则由于亚甲基的贫电子性不容易发生 $\beta$-氢消除得到相应的醛. 在第二个过程中, 富电子取代 的芳香醛由于羰基的亲电性被削弱从而不容易被醇亲 核进攻得到半缩醛, 因此不能够顺利得到最终的酯, 相 反对于贫电子取代的芳香醛则可以很顺利与醇反应得 到半缩醛，进而发生 $\beta$-氢消除得到最终的酯.

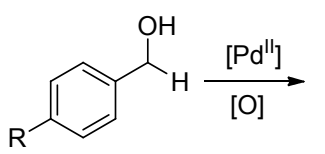

the first step<smiles>[R]OC(=O)c1ccc([R])cc1</smiles>

图式 11 氧化酯化反应的可能反应历程

Scheme 11 Possible pathway for the oxidative esterification reaction

基于以上分析, 我们推测: 使用相对较为贫电子的
钯源不仅可以使之容易与贫电子芐醇配位加速 $\beta$-氢消 除得到相应的醛，而且还可以活化原位产生的富电子芳 香醛使之容易与醇反应得到半缩醛中间体，并发生之后 的 $\beta$-氢消除得到相应的酯，从而可以解决因底物电子效 应所带来的产率低的问题. 正如我们所料，当往反应体 系里面加入四氟硼酸银从而可以原位生成贫电子的四 氟嗍酸钯物种时, 无论是富电子还是贫电子取代的茮醇 都可以很好地转化为相应的酯, 产率中等到优秀. 另外, 在对脂肪族醇类的底物扩展过程中发现，配体对该类醇 类的转化起到了重要的作用，使用我们实验室之前开发 的贫电子的磷烯烃配体 ${ }^{[63]}$ 便可以使这些醇类转化为相 应的氧化酯化产物，产率中等.

钯催化的醇氧化成醛的反应属于合成化学里面的 基础研究领域 ${ }^{[64]}$. 迄今为止，尽管对过渡金属催化的醇 氧化成醛的反应有众多报道，但是对其中存在的基本科 学问题的理解仍知之甚少，例如如何控制反应的选择性 使其停止在醛的生成或者继续与醇发生氧化酯化反应、 配体在该类反应中所起的关键作用等等，找到其中的关 键因素对于指导我们化学合成以及设计反应具有重要 意义 ${ }^{[65]}$. 基于我们对醇的氧化交叉酯化反应的理解以 及对醛与醇氧化酯化反应的研究兴趣, 2012 年, 我们 ${ }^{[66]}$ 报道了共价偶联配体茮基促进的钯催化的醛与醇的选 择性氧化酯化反应(Eq. 19), 发现氧化剂可以很好的控 制该类反应的选择性 ${ }^{[67]}$. 我们对其中的一些科学问题 做了比较详细的研究和讨论.

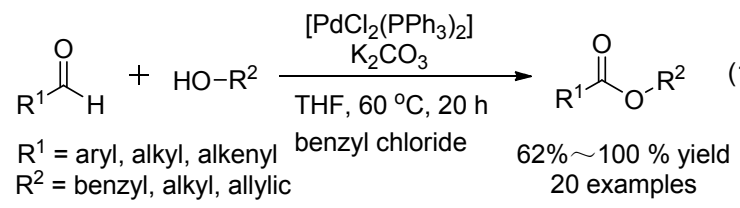

要认识和理解这些科学问题, 首先需要先从反应的 机理上对醇氧化成醛反应以及醛与醇氧化酯化反应进 行区分. 我们推测它们的反应机理如下: 首先是氧化剂 $\mathrm{A}-\mathrm{B}$ 对低价金属钯进行氧化加成得到中间体 $\mathbf{I}$, 其后醇 在碱作用下对中间体 I 进行醇解得到中间体 II, 此时中 间体 II 可以通过 $\beta$-氢消除途径得到醛并释放出钯氢物 种 III (Path I), III 经过还原消除重新得到零价钯物种实 现醇氧化到醛的催化循环．中间体 II 也可以通过醛的插 入途径得到半缩醛中间体 IV (Path II), 中间体 IV 再经 过 $\beta$-氢消除途径即得到酯并释放钯氢物种 III, III 则经 过还原消除重新得到零价钯物种实现醛与醇氧化酯化 的催化循环(Scheme 12). 从反应机理上不难看出: 要想 高选择性地实现醛醇的氧化酯化反应，关键就在于控制 醇氧化成醛相对于生成半缩醛钯中间体物种的反应速 度. 而在这个过程中 $\mathrm{A}-\mathrm{Pd}$ 结构始终存在于催化循环里 
面, 我们推测 $\mathbf{A}$ 作为共价偶联配体, 其结构和电性对反 应路径的选择起着十分关键的作用.

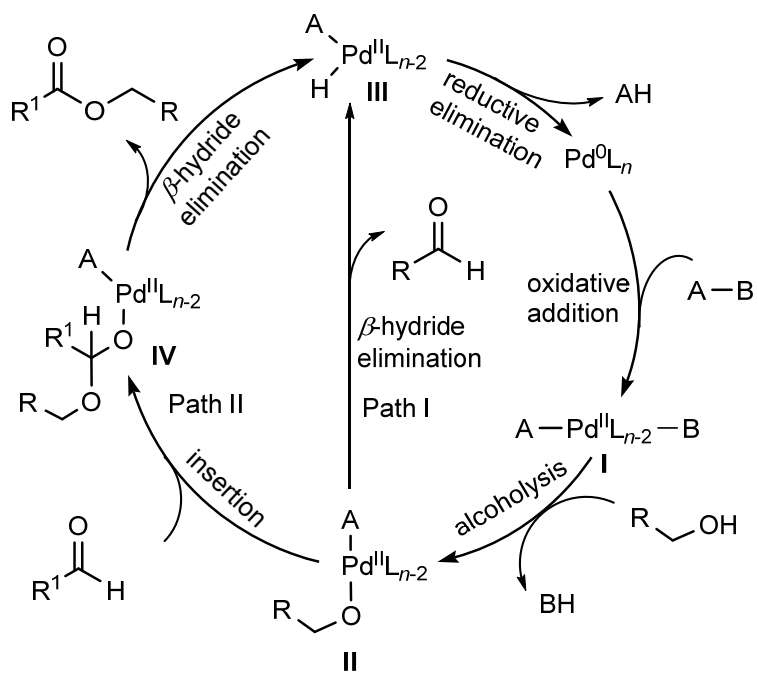

图式 12 醇氧化成醛(Path I)和醇与醛氧化酯化反应(Path II) 的可能催化循环

Scheme 12 Proposed catalytic cycles for alcohol oxidation (Path I) and oxidative esterification (Path II)

根据许多相关文献报道可知, 有机卤代物 $\mathrm{R}-\mathrm{X}$ 可以作 为氧化剂对零价钯进行氧化加成得到 $R-P d-X$, 随后 $\mathrm{Pd}-\mathrm{X}$ 键会优先发生醇解得到 $\mathrm{R}-\mathrm{Pd}$ 键保留的物种 ${ }^{[78]}$, 此时有机骨架 $\mathrm{R}$ 通过碳钯键作为共价偶联配体与钯相 互作用. 为了验证我们推测的共价偶联配体对反应选择 性的影响, 首先我们选择了含有不同 $\mathrm{R}$ 基团的有机卤代 物作为氧化剂对苯甲醛与对甲基苄醇的氧化酯化反应 进行研究, 结果发现只有茮氯作为氧化剂可以得到氧化 酯化产物 3a， $\alpha$-氯代苯乙酮以及对溴甲苯则选择性地发 生了醇的氧化成醛反应(表 1), 这说明氧化剂一定程度 上控制了该类反应的选择性.

根据相关文献报道, 在反应过程中, 芐氯与钯氧化 加成得到的钯物种会选择性的在 $\mathrm{Pd}-\mathrm{Cl}$ 键上发生醇 解 ${ }^{[69]}$, 最终苄基作为共价偶联配体得以保留(Eq. 20), 而 $\alpha$-氯代苯乙酮与钯氧化加成得到的钯物种会选择性 地在烯醇化后的 $\mathrm{Pd}-\mathrm{O}$ 键上发生醇解，最终得到氯原子 得以保留的钯物种(Eq. 21), 对溴甲苯与钯氧化加成得 到的钯物种则会选择性的在地在 $\mathrm{Pd}-\mathrm{Br}$ 键上发生醇 解 ${ }^{[70]}$, 最终得到对甲基苯基得以保留的钯物种(Eq. 22). 这些结果说明苄基作为共价偶联配体在选择性醛醇氧 化酯化反应中起着关键作用.

为了进一步验证苄基作为共价偶联配体的关键作 用, 我们还使用两种不同的钯催化剂前体 $\mathrm{Bn}[\mathrm{PdCl}-$ $\left.\left(\mathrm{PPh}_{3}\right)_{2}\right]$ 和 $\left[\mathrm{PdCl}_{2}\left(\mathrm{PPh}_{3}\right)_{2}\right]$ 做了当量实验. 结果显示: 没有 芐基共价偶联配体的 $\left[\mathrm{PdCl}_{2}\left(\mathrm{PPh}_{3}\right)_{2}\right]$ 体系只发生了醇氧
表 1 钯催化氧化酯化反应中三种氧化剂的测试效果

Table 1 Three oxidants tested in palladium-catalyzed oxidative esterification

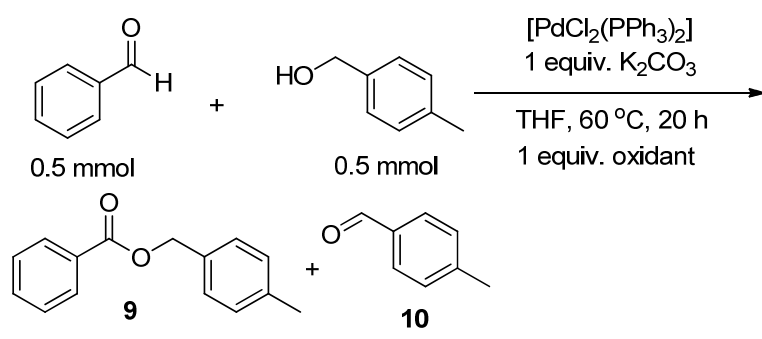

\begin{tabular}{ccc}
\hline Oxidant & \multicolumn{2}{c}{ Yield $^{a} / \%$} \\
\cline { 2 - 3 } & $93\left(96^{b}\right)$ & $\mathbf{9 0}$ \\
\hline
\end{tabular}

${ }^{a}$ GC-Yield. ${ }^{b}$ Isolated yield.

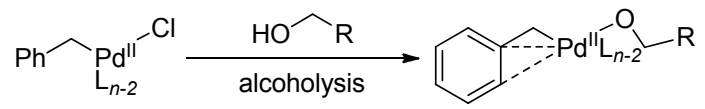

$$
\begin{aligned}
& A=\text { benzyl }
\end{aligned}
$$

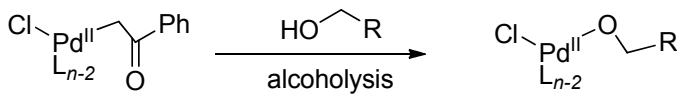

$$
\begin{aligned}
& \mathrm{A}=\mathrm{Cl}
\end{aligned}
$$

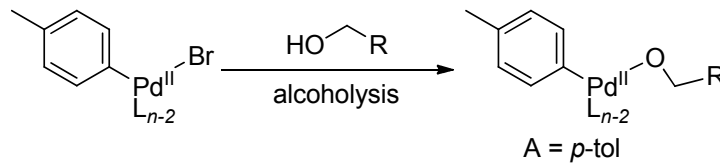

化成醛的反应(Eq. 23), 而氧化酯化反应在 $\mathrm{Bn}[\mathrm{PdCl}-$ $\left(\mathrm{PPh}_{3}\right)_{2}$ ]催化下可以顺利进行(Eq. 24), 这些结果都说明 茮基作为共价偶联配体很好的控制了反应的选择性，使 得氧化酯化反应顺利进行.

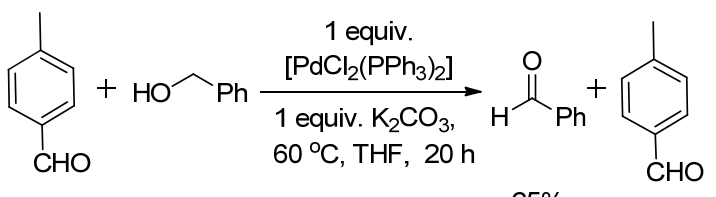<smiles>Cc1ccc(C=O)cc1</smiles>

既然如此，共价偶联配体是如何影响反应选择性的 呢? 我们推测苄基作为共价偶联配体与钯可以很容易 地发生 $\eta^{3}$ 配位，该配位效应使钯上的配体如三苯基膦较 
容易发生配体解离, 从而促进了醛与钯的配位, 利于半 缩醛中间体的生成. 为了证明该 $\eta^{3}$ 配位效应，我们往该 体系里面加入双齿鳌合配体 1,1'-双(二苯基膦)二茂铁 (dppf), 结果导致氧化酯化产物大大降低的同时还伴有 少量的醇氧化成醛产物, 这可能就是因为双齿螯合配体 dppf 与钯强烈的螯合作用阻碍了 $\eta^{3}$ 配位的形成, 从而使 反应不能很顺利的进行, 这也验证了我们上面推断的合 理性.

我们使用苄氯作为氧化剂很好地实现了醛醇的氧 化成酯反应，不仅克服了醛醇氧化酯化反应中往往因使 用过强的氧化剂而导致反应选择性差的缺陷, 而且芐氯 作为廉价易得的工业品, 其最终副产物只有甲苯, 便于 后处理, 增加了反应的实用性. 难能可贵的是该反应使 用的底物以及氧化剂的比例是 $1: 1$ 的关系, 原子经济 性优势明显, 底物的普适性也很好, 不论贫电子取代还 是富电子取代、芳香族还是脂肪族的醛醇都能够顺利反 应并得到中等及以上的收率. 更让人欣喜的是, 该反应 还可以在无溶剂条件下仅使用 $0.25 \mathrm{~mol} \%$ 的钯催化剂就 可以使反应很好的进行.

\section{4 碳杂成键的氧化偶联反应}

苯并噻唑作为制药领域中重要的一类杂环化合 物 ${ }^{[71]}$, 其通常的合成方法往往需要往反应体系里面加 入一些非环境友好的氧化剂如高价碘化合物、溴、醌类 等 ${ }^{[72]}$, 或者是使用一些功能团化的卤代物作为起始原 料 ${ }^{[73]}$, 这些都在一定程度上限制了这些方法的实用性. 相比之下, 通过直接的氧化偶联反应实现苯并噻唑类化 合物的合成吸引了众多化学工作者. 2012 年, 我们 ${ }^{[74]}$ 实 现了铁催化的通过氧化碳氢键功能化反应实现苯并噻 唑类化合物的合成(Eq. 25). 该反应使用廉价易得且低 毒性的三氯化铁作为催化剂, 规避了碳氢功能化反应中 常常使用贵金属作为催化剂的缺陷 ${ }^{[75]}$, 而且使用了廉 价的过硫酸钠作为氧化剂, 反应条件温和. 同时该反应 对于 $N$-烷基取代的硫酰胺以及硫嫝化合物也可以顺利 反应得到相应的目标产物.

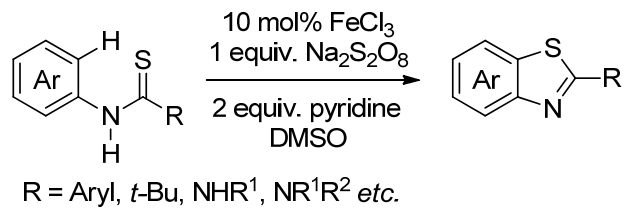

我们对该反应的机理也做了一些探究: (1)邻位的同 位素标记实验得到动力学同位素效应(KIE)值为 1.3 (Eq. 26), 这说明碳氢键的断裂过程不是反应的决速步. (2)在 反应中添加自由基捕获剂四甲基哌啶氮氧化物 (TEMPO), 反应产率大大降低, 这说明该反应可能历经 自由基过程(Eq. 27). (3)在线红外跟踪发现：碳氢键的活
化以及碳硫键的形成需要在催化剂、氧化剂以及底物共 存在的情况下才会发生(图 4). (4)进一步的动力学实验 测得该反应的反应速率对底物浓度呈现一级关系, 对氧 化剂浓度则是零级关系.
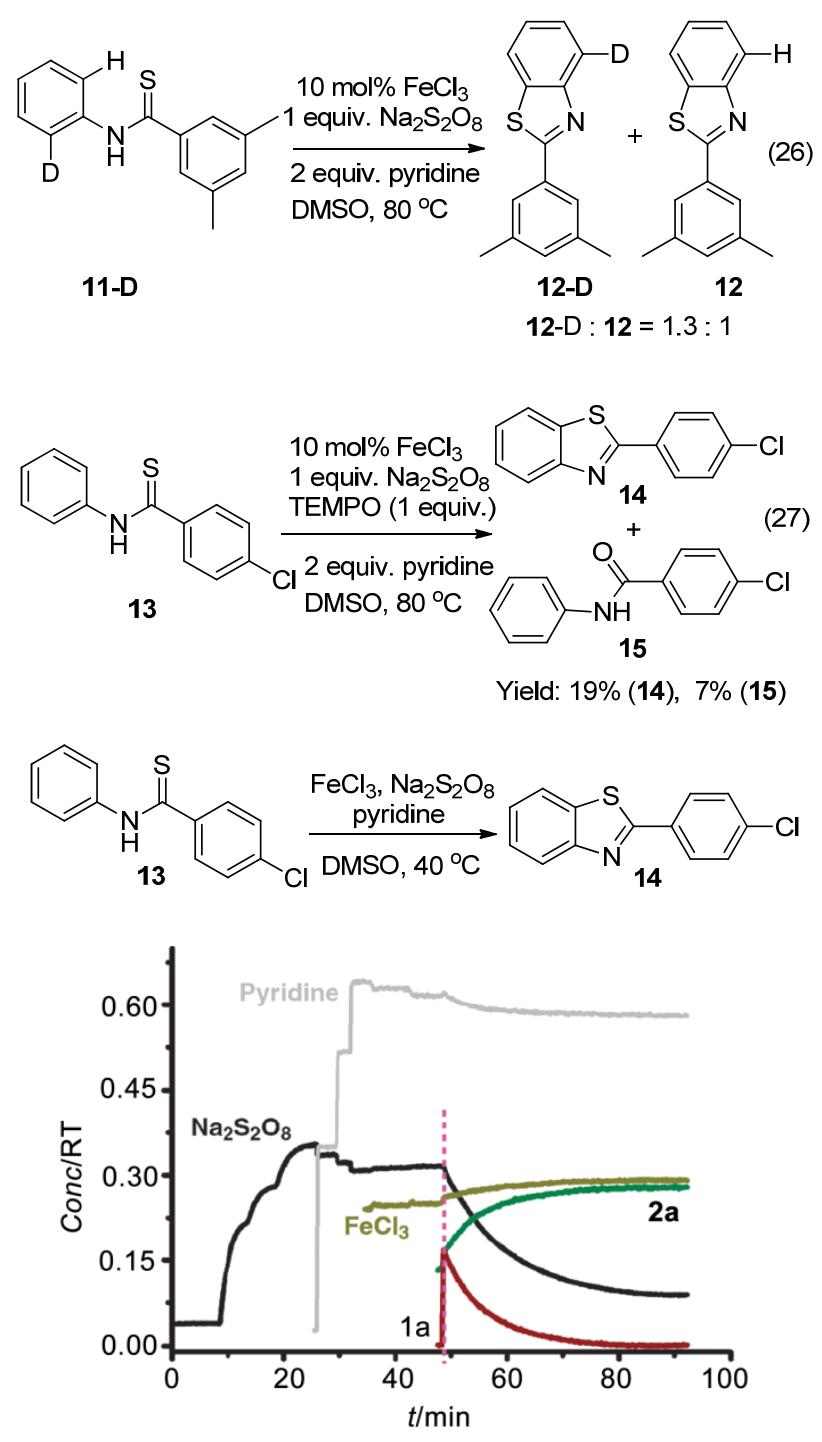

图 $440{ }^{\circ} \mathrm{C}$ 条件下 $\mathrm{Na}_{2} \mathrm{~S}_{2} \mathrm{O}_{8}(0.2 \mathrm{~mol} / \mathrm{L})$ 、吡啶 $(4 \mathrm{~mol} / \mathrm{L}), \mathrm{FeCl}_{3}$ $(0.02 \mathrm{~mol} / \mathrm{L})$ 和 $1 \mathrm{a}(0.2 \mathrm{~mol} / \mathrm{L})$ 依次加入到 $5 \mathrm{~mL} \mathrm{DMSO}$ 中通过 在线红外检测得到二维动力学图

Figure 4 The 2D-kinetic profiles of the stoichiometric reaction of $\mathrm{Na}_{2} \mathrm{~S}_{2} \mathrm{O}_{8}(0.2 \mathrm{~mol} / \mathrm{L})$, pyridine $(4 \mathrm{~mol} / \mathrm{L}), \mathrm{FeCl}_{3}(0.02 \mathrm{~mol} / \mathrm{L})$ and $1 \mathrm{a}(0.2 \mathrm{~mol} / \mathrm{L})$ added to $5 \mathrm{~mL} \mathrm{DMSO}$ at $40{ }^{\circ} \mathrm{C}$ one by one, and the reaction was monitored by in situ IR

根据上述信息，我们提出了如下的反应机理： $N$-苯 基苯硫酰胺在吡啶辅助下被三价铁氧化失去一个单电 子得到硫自由基 $\mathbf{A}$ (Path A). 与此同时, 铁被还原到二 价再被过硫酸钠氧化到三价实现催化循环. 接着硫自由 基对芳环进行自由基亲电加成，之后再被过硫酸钠氧化 实现芳构化得到目标产物 B. 吡啶在整个反应过程中可 
能起到了捕获体系中产生的氢离子的作用，从而抑制了 $N$-苯基苯硫酰胺分解成 $N$-苯基苯甲酰胺的过程(Path B) (Scheme 13).

除了碳硫氧化成键以外, 我们还报道了碳氧氧化成 键反应(Eq. 28) ${ }^{[76]}$. 迄今为止, 使用 $\mathrm{O}-\mathrm{H}$ 物种实现氧化 碳氧成键的报道仍然非常稀少 ${ }^{[77]}$. 与一般的交叉脱氢 偶联反应(CDC 反应)不同 ${ }^{[78]}$, 我们直接使用茮基碳氢化 合物种作为反应底物, 不需要邻位杂原子的活化, 解决 了该类底物的碳氢键由于失去杂原子活化往往存在的 反应活性低、选择性差、难于与过渡金属配位而很难被 活化的问题 ${ }^{[79]}$. 该反应还可以使用亚催化量的二氯二 氭基苯醌(DDQ) 以及廉价低毒性的二氧化锰 ${ }^{[80]}$ 作为再 生氧化剂即可高收率地得到目标产物, 避免了使用化学 计量昂贵且具有中等毒性的二氯二氭基苯醌(DDQ)作 为氧化剂, 降低了成本的同时也使反应更为绿色经济. 反应底物的适用范围也较广, 不论富电子还是贫电子取 代的二苯甲烷类化合物都能很好地转化为相应的产物. 需要指出的是, 含有硝基取代的二苯甲烷没有得到相应 的产物，说明该反应可能经过了一个自由基的过程，因 为硝基往往可以抑制自由基反应. 不同的长链酸、芳香 酸、烯基酸都能很好地参与反应. 而对于酸性较弱的羧 酸类化合物, 由于他们的反应活性较低, 需要往体系里 加入酸性强、亲核性弱的三氟乙酸作为添加剂, 以此来 提高二氧化锰再生 DDQ 的能力, 从而促使反应高效进 行.

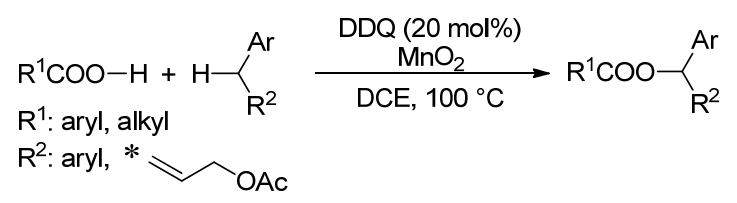

该反应还可以在室温下实现活性较高的苯丙烯的 双乙酰氧基化反应(Scheme 14), 该双乙酰氧基化产物 19 经过 Tsuji-Trost 反应即可得到二芳基化产物 20, 该二 芳基化产物可以在室温下利用水作为亲核试剂实现碳 氧成键的氧化偶联反应, 反应得到的中间产物醇在该条
件下发生氧化反应最终得到了酮产物 21. 我们推测该 反应首先是苯丙烯在强氧化剂二氯二氰基苯醌(DDQ) 存在下氧化成碳正离子 17 , 之后 17 接受醋酸根的亲核 进攻得到单乙酰氧基化产物 18, 再发生一次类似的碳 氧氧化成键反应即得到双乙酰氧基化产物 19 . 由于单 乙酰氧基化产物 18 中的亚甲基旁边连有氧原子, 其在 二氯二氰基苯醌(DDQ)存在的情况下反应活性非常高, 因此，我们没有分离出中间体单乙酰氧基化产物 18. 为 了验证推测过程的合理性，我们合成了单乙酰氧基化产 物 18 并将其顺利的应用到了反应体系中，与不同羧酸 的碳氧氧化偶联产物的收率在中等以上.

为了进一步研究该反应可能的反应机理, 我们往反 应体系里面分别加入自由基捕获剂 TEMPO 或 1,1-二苯 乙烯(Eqs. 29，30), 结果均导致该反应产率大大降低甚 至被彻底抑制，说明该反应可能经历了自由基的过程. 该反应的分子间以及分子内的同位素标记实验得到的 KIE 值分别为 4.8 和 1.6 (Eq. 31, 32), 说明碳氢键断裂的 过程可能是反应的决速步.

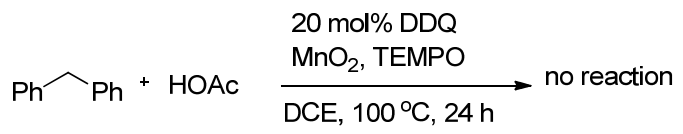

$$
\begin{aligned}
& 20 \mathrm{~mol} \% \mathrm{DDQ}, \mathrm{MnO}_{2}
\end{aligned}
$$

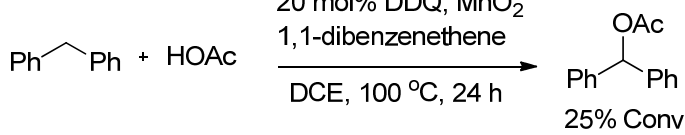

$$
\begin{aligned}
& 25 \% \text { yield }
\end{aligned}
$$

基于以上信息，我们提出可能的反应机理如 Scheme 15 所示: 该反应由 DDQ 引发经过单电子氧化过 程(SET)使得二苯甲烷转化为茮基自由基中间体 $\mathbf{A}$, 随 后，其再被进一步氧化得到碳正离子中间体 $\mathbf{B}$ ，并接受 亲核试剂羧酸的进攻得到碳氧氧化偶联反应产物 $\mathbf{C}$ 的 同时释放出 $\mathrm{DDQH}_{2}, \mathrm{DDQH}_{2}$ 经二氧化锰氧化再释放出 DDQ 实现催化循环.

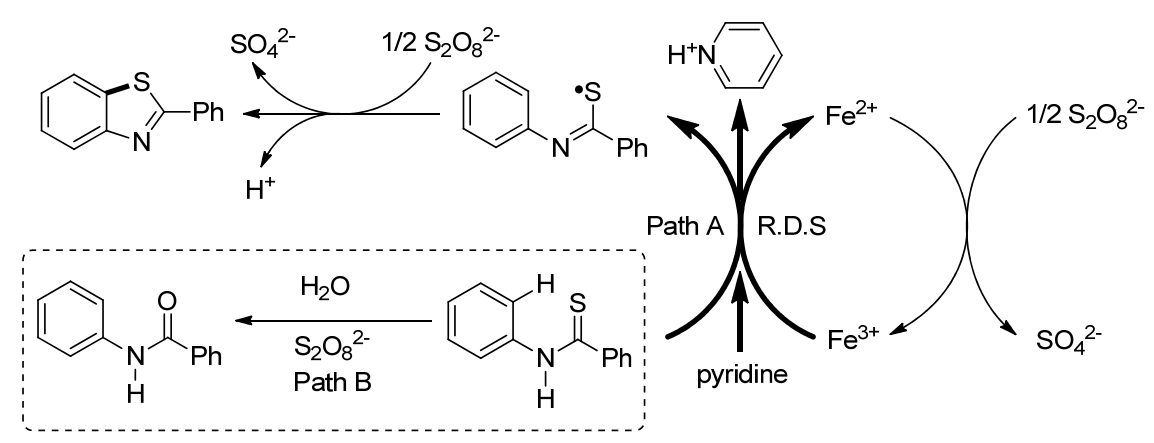

图式 13 铁催化氧化 $\mathrm{C}-\mathrm{H}$ 键官能团化构建 $\mathrm{C}-\mathrm{S}$ 键反应的可能机理

Scheme 13 Proposed mechanism of Fe-catalyzed oxidative $\mathrm{C}-\mathrm{H}$ functionalization/ $\mathrm{C}-\mathrm{S}$ bond formation 


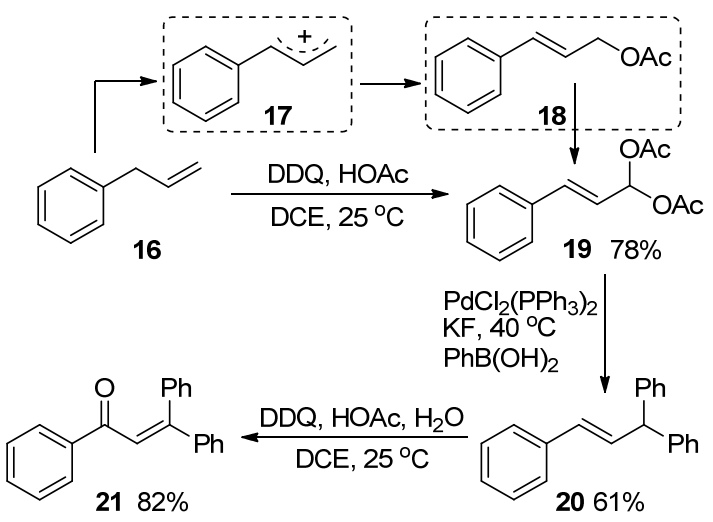

图式 $14 \mathrm{DDQ}$ 促进的苯丙烯和乙酸的碳氧偶联反应的可能反 应历程和三取代烯烃的合成方法

Scheme 14 DDQ-mediated oxidative $\mathrm{C}-\mathrm{O}$ coupling of allylbenzene with acetic acid and the synthetic routes toward the trisubstituted olefin
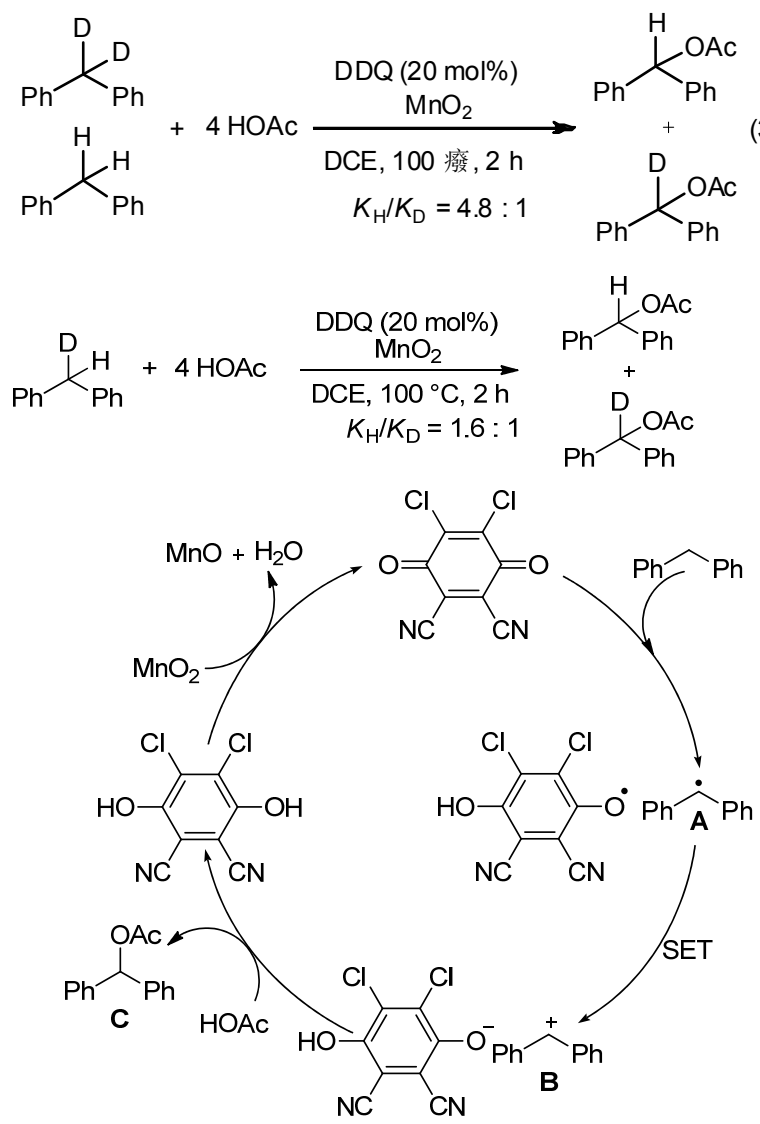

图式 $15 \mathrm{DDQ}$ 促进的 $\mathrm{C}_{\mathrm{sp}}{ }^{3}-\mathrm{H}$ 键和羧酸化合物的碳氧氧化成 键反应的可能机理

Scheme 15 Proposed mechanism of DDQ-catalyzed oxidative $\mathrm{C}-\mathrm{O}$ coupling of $\mathrm{C}_{\mathrm{sp}^{3}}{ }^{3}-\mathrm{H}$ bonds with carboxylic acids

\section{3 结论与展望}

综上所述, 顺应有机化学发展的规律以及社会发展 的需求, 我们从两种金属试剂作为亲核试剂的第一代氧 化偶联反应发展到两种碳氢化合物作为亲核试剂的第
三代氧化偶联反应，反应的原子经济性一步步提高，反 应条件也更为温和，这些方法学的进展为一些天然产物 和生理活性分子的构建提供了新思路，也为氧化偶联反 应的发展及其未来发展趋势提供了一些线索和启示. 近 年来, 氧化偶联反应受到了越来越多的化学家以及科研 工作者的关注, 发展十分迅速, 已经渐渐成为有机合成 化学中构筑各种化学键的强有力的工具之一, 但是作为 有机合成化学里面仍然比较年轻的领域, 氧化偶联反应 的发展仍然面临着诸多的挑战. (1)如何高选择性地实现 氧化交叉偶联反应是其挑战之一. 尽管通过引入不同的 定位基团或者使用数学选择性控制方面取得了一定的 成果，但是这也在一定程度上限制了底物的适用范围以 及其潜在的实用价值. (2)多数的氧化偶联反应都不可避 免地使用了贵金属如钯、铑、金、钉等作为催化剂, 如 何开发廉价低毒的金属催化体系以及非金属催化体系, 提高反应的原子转化数( TON)等方面也将是其面临的挑 战之一. (3)氧化剂在氧化偶联反应中扮演着至关重要的 角色, 发展一系列使用氧气或过氧化氢等绿色环保的氧 化剂代替现有的有机氧化剂以及无机氧化剂将会是更 为理想的选择. (4)目前, 氧化偶联反应还缺乏成体系的 理论指导, 相关的动力学研究以及对反应机理的理解方 面还有待进一步的深入研究.

\section{作者简介}

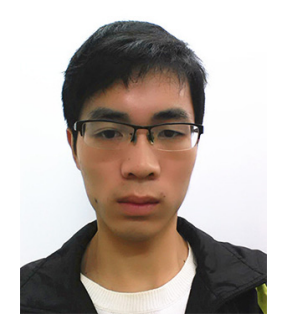

张剑, 2009 年考入武汉大学化学与分子科学学院, 2011 年进入雷爱文教授课题组学习开展课题研究, 并 在雷爱文教授的指导下完成本科毕业论文设计. 2013 年 保送雷爱文教授的硕士研究生, 主要从事氧气活化和自 由基脱羧偶联的反应研究.

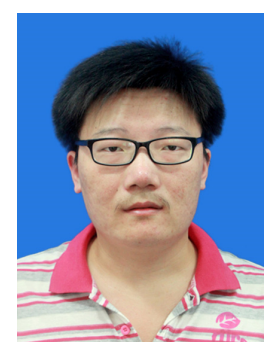

陆庆全，博士. 2014 年 12 月毕业于武汉大学，获得 理学博士学位，师从雷爱文教授，主要从事氧气引发或 
参与的自由基氧化偶联反应及相关机理研究.

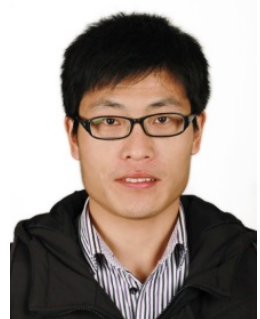

刘超, 博士. 2012 年毕业于武汉大学, 获得理学博 士学位, 师从雷爱文教授. 曾于 2008 年 10 月至 2009 年 10 月间, 在中国国家留学基金管理委员会的支持下赴 英国杜伦大学 Todd B. Marder 教授课题组交流学习. 现 主要从事钯催化和镍催化的偶联反应以及光电功能高 分子的方法学合成等领域的探究.

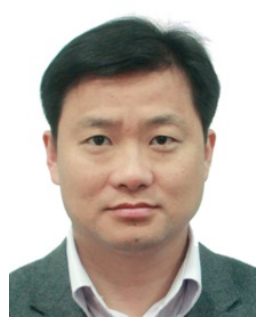

雷爱文, 教授. 1995 年本科毕业于安徽淮北煤炭师 范学院, 2000 年于中国科学院上海有机化学研究所获理 学博士学位, 师从陆熙炎院士. 2000 年至 2003 年间, 在 美国宾夕法尼亚州州立大学张绪暮教授课题组从事博 士后研究. 2003 年至 2005 年在美国斯坦福大学 James P. Collman 教授课题组从事研究助理工作. 2005 年回国到 武汉大学化学与分子科学学院任教, 成为全职教授. 2010 年获得国家杰出青年基金, 2014 年入选长江学者特 聘教授. 现主要从事有机小分子合成方法学以及化学反 应活性中间体和反应动力学的机理研究

\section{References}

[1] Negishi, E.-I.; de Meijere, A. Handbook of Organopalladium Chemistry for Organic Synthesis, Wiley-Interscience, New York, 2002.

[2] (a) Heck, R. F.; Nolley, J. P. J. Org. Chem. 1972, 37, 2320.

(b) Miyaura, N.; Suzuki, A. J. Chem. Soc., Chem. Commun. 1979, 866.

(c) Milstein, D.; Stille, J. K. J. Am. Chem. Soc. 1979, 101, 4992.

(d) Baba, S.; Negishi, E. J. Am. Chem. Soc. 1976, 98, 6729.

(e) Hatanaka, Y.; Hiyama, T. J. Org. Chem. 1988, 53, 918.

(f) Guram, A. S.; Rennels, R. A.; Buchwald, S. L. Angew. Chem., Int. Ed. 1995, 34, 1348.

[3] (a) Liu, C.; Zhang, H.; Shi, W.; Lei, A. Chem. Rev. 2011, 111, 1780. (b) Liu, Q.; Zhang, H.; Lei, A. Angew. Chem., Int. Ed. 2011, 50, 10788.

(c) Shi, W.; Liu, C.; Lei, A. Chem. Soc. Rev. 2011, 40, 2761.

(d) Liu, C.; Jin, L. Synlett 2010, 2527.
[4] Lipshutz, B. H.; Siegmann, K.; Garcia, E.; Kayser, F. J. Am. Chem. Soc. 1993, 115, 9276

[5] Zhao, Y.; Wang, H.; Hou, X.; Hu, Y.; Lei, A.; Zhang, H.; Zhu, L. J. Am. Chem. Soc. 2006, 128, 15048.

[6] Jin, L.; Zhao, Y.; Zhu, L.; Zhang, H.; Lei, A. Adv. Synth. Catal. 2009, 351, 630 .

[7] Oi, S.; Fukita, S.; Inoue, Y. Chem. Commun. 1998, 2439

[8] Zhao, Y.; Jin, L.; Li, P.; Lei, A. J. Am. Chem. Soc. 2008, 130, 9429.

[9] (a) Luh, T.; Leung, M.; Wong, K. Chem. Rev. 2000, 100, 3187. (b) Woell, J. B.; Fergusson, S. B.; Alper, H. J. Org. Chem. 1985, 50, 2134.

[10] Frisch, A. C.; Beller, M. Angew. Chem., Int. Ed. 2005, 44, 674.

[11] Liu, Q.; Li, G.; He, J.; Liu, J.; Li, P.; Lei, A. Angew. Chem., Int. Ed. 2010, 49, 3371.

[12] Yoshida, H.; Yamaryo, Y.; Ohshita, J.; Kunai, A. Tetrahedron Lett. 2003, 44, 1541.

[13] (a) Inoue, S.; Shiota, H.; Fukumoto, Y.; Chatani, N. J. Am. Chem. Soc. 2009, 131, 6898.

(b) Guan, Z.; Ren, Z.; Spinella, S. M.; Yu, S.; Liang, Y.; Zhang, X. J. Am. Chem. Soc. 2008, 131, 729.

(c) Giri, R.; Yu, J. J. Am. Chem. Soc. 2008, 130, 14082.

[14] (a) Inoue, S.; Shiota, H.; Fukumoto, Y.; Chatani, N. J. Am. Chem. Soc. 2009, 131, 6898.

(b) Guan, Z. H.; Ren, Z. H.; Spinella, S. M.; Yu, S. C.; Liang, Y. M.; Zhang, X. M. J. Am. Chem. Soc. 2009, 131, 729.

(c) Giri, R.; Yu, J. Q. J. Am. Chem. Soc. 2008, 130, 14082.

(d) Orito, K.; Horibata, A.; Nakamura, T.; Ushito, H.; Nagasaki, H.; Yuguchi, M.; Yamashita, S.; Tokuda, M. J. Am. Chem. Soc. 2004, 126, 14342.

(e) Zhao, Y.; Jin, L.; Li, P.; Lei, A. J. Am. Chem. Soc. 2008, 130, 9429.

[15] (a) Eckhardt, M.; Fu, G. C. J. Am. Chem. Soc. 2003, 125, 13642.

(b) Altenhoff, G.; Wuertz, S.; Glorius, F. Tetrahedron Lett. 2006, 47, 2925.

(c) Cahiez, G.; Gager, O.; Buendia, J. Angew. Chem., Int. Ed. 2010, 49, 1278.

[16] Chen, M.; Zheng, X.; Li, W.; He, J.; Lei, A. J. Am. Chem. Soc. 2010, 132, 4101 .

[17] Izawa, Y.; Shimizu, I.; Yamamoto, A. Bull. Chem. Soc. Jpn. 2004, 77, 2033.

[18] Murata, S.; Teramoto, K.; Miura, M.; Nomura, M. J. Chem. Res., Synop. 1993, 434.

[19] Fuchita, Y.; Utsunomiya, Y.; Yasutake, M. J. Chem. Soc., Dalton Trans. 2001, 2330.

[20] Dick, A. R.; Hull, K. L.; Sanford, M. S. J. Am. Chem. Soc. 2004, 126,2300

[21] (a) Kamata, K.; Yamaguchi, S.; Kotani, M.; Yamaguchi, K.; Mizuno, N. Angew. Chem., Int. Ed. 2008, 47, 2407.

(b) Eglinton, G.; Galbraith, A. R. Chem. Ind. 1956, 737.

(c) Hay, A. S. J. Org. Chem. 1960, 25, 1275.

(d) Hay, A. S. J. Org. Chem. 1962, 27, 3320.

(e) Ranu, B. C.; Banerjee, S. Lett. Org. Chem. 2006, 3, 607.

(f) Jiang, H.-F.; Tang, J.-Y.; Wang, A. Z.; Deng, G.-H.; Yang, S.-R. Synthesis 2006, 1155.

(g) Yadav, J. S.; Reddy, B. V. S.; Reddy, K. B.; Gayathri, K. U.; Prasad, A. R. Tetrahedron Lett. 2003, 44, 6493.

(h) Nishihara, Y.; Ikegashira, K.; Hirabayashi, K.; Ando, J.-i.; Mori, A.; Hiyama, T. J. Org. Chem. 2000, 65, 1780.

(i) Siemsen, P.; Livingston, R. C.; Diederich, F. Angew. Chem., Int. Ed. 2000, 39, 2632.

[22] (a) Glaser, C. Ber. Dtsch. Chem. Ges. 1869, 2, 422.

(b) Glaser, C. Ann. Chem. Pharm. 1870, 154, 137. 
(c) Yang, F.; Cui, X.; Li, Y.-N.; Zhang, J.; Ren, G.-R.; Wu, Y. Tetrahedron 2007, 63, 1963.

(d) Shi, M.; Qian, H.-X. Appl. Organomet. Chem. 2006, 20, 771.

(e) Rossi, R.; Carpita, A.; Bigelli, C. Tetrahedron Lett. 1985, 26, 523.

(f) Qian, M.; Negishi, E. Org. Process Res. Dev. 2003, 7, 412.

(g) Lei, A.; Srivastava, M.; Zhang, X. J. Org. Chem. 2002, 67, 1969.

(h) Chalifoux, W. A.; Ferguson, M. J.; Tykwinski, R. R. Eur. J. Org. Chem. 2007, 1001.

(i) Yan, J.; Lin, F.; Yang, Z. Synthesis 2007, 1301.

[23] (a) Shun, A. L. K. S.; Tykwinski, R. R. Angew. Chem., Int. Ed. 2006, 45, 1034.

(b) Haley, M. M.; Tykwinski, R. R. Carbon-Rich Compounds: From Molecules to Materials, Wiley-VCH, Weinheim, 2006.

(c) Diederich, F.; Stang, P. J.; Tykwinski, R. R. Acetylene Chemistry: Chemistry, Biology and Material Science, Wiley-VCH, Weinheim, 2005.

[24] (a) Kim, S.; Kim, S.; Lee, T.; Ko, H.; Kim, D. Org. Lett. 2004, 6, 3601 .

(b) Nishihara, Y.; Ikegashira, K.; Mori, A.; Hiyama, T. Tetrahedron Lett. 1998, 39, 4075.

(c) Montierth, J. M.; DeMario, D. R.; Kurth, M. J.; Schore, N. E. Tetrahedron 1998, 54, 11741.

(d) Alami, M.; Ferri, F. Tetrahedron Lett. 1996, 37, 2763.

[25] Yin, W.; He, C.; Chen, M.; Zhang, H.; Lei, A. Org. Lett. 2008, 11, 709.

[26] (a) Matsumoto, F.; Chujo, Y. Pure Appl. Chem. 2006, 78, 1407.

(b) Butler, P.; Gallagher, J. F.; Manning, A. R.; Mueller-Bunz, H.; McAdam, C. J.; Simpson, J.; Robinson, B. H. J. Organomet. Chem. 2005, 690, 4545.

(c) McAdam, C. J.; Manning, A. R.; Robinson, B. H.; Simpson, J. Inorg. Chim. Acta 2005, 358, 1673.

(d) Gallagher, J. F.; Butler, P.; Hudson, R. D. A.; Manning, A. R. J. Chem. Soc., Dalton Trans. 2002, 75.

(e) Butler, P.; Gallagher, J. F.; Manning, A. R. Inorg. Chem. Commun. 1998, 1,343 .

(f) Onitsuka, K.; Tao, X. Q.; Sonogashira, K. Bull. Chem. Soc. Jpn. 1994, 67, 2611.

(g) Matsumoto, M.; Kuroda, K. Tetrahedron Lett. 1980, 21, 4021.

(h) Miyano, S.; Izumi, Y.; Hashimoto, H. J. Chem. Soc., Chem. Commun. 1978, 446.

[27] (a) Lanci, M. P.; Brinkley, D. W.; Stone, K. L.; Smirnov, V. V.; Roth, J. P. Angew. Chem., Int. Ed. 2005, 44, 7273.

(b) Kieber-Emmons, M. T.; Riordan, C. G. Acc. Chem. Res. 2007, 40,618 .

[28] (a) Smith, E. H.; Whittall, J. Organometallics 1994, 13, 5169.

(b) Klein, H. F.; Beck-Hemetsberger, H.; Reitzel, L.; Rodenhaeuser, B.; Cordier, G. Chem. Ber. 1989, 122, 43.

[29] (a) Kamata, K.; Yamaguchi, S.; Kotani, M.; Yamaguchi, K.; Mizuno, N. Angew. Chem., Int. Ed. 2008, 47, 2407.

(b) Siemsen, P.; Livingston, R. C.; Diederich, F. Angew. Chem., Int. Ed. 2000, 39, 2632.

[30] Liu, Q.; Li, G.; He, J.; Liu, J.; Li, P.; Lei, A. Angew. Chem., Int. Ed. 2010, 49, 3371.

[31] (a) Belmont, P. In Silver in Organic Chemistry, Ed.: Harmata, M., John Wiley \& Sons Inc., Hoboken, NJ, 2010, Chapter 5, pp. 143 165.

(b) Alvarez-Corral, M.; Munoz-Dorado, M.; Rodriguez-Garcia, I. Chem. Rev. 2008, 108, 3174.

(c) Weibel, J. M.; Blanc, A.; Pale, P. Chem. Rev. 2008, 108, 3149

[32] (a) Hou, X. L.; Cheung, H. Y.; Hon, T. Y.; Kwan, P. L.; Lo, T. H.;
Tong, S. Y.; Wong, H. N. C. Tetrahedron 1998, 54, 1955.

(b) Kirsch, S. F. Org. Biomol. Chem. 2006, 4, 2076.

(c) Lipshutz, B. H. Chem. Rev. 1986, 86, 795.

(d) Keay, B. A. Chem. Soc. Rev. 1999, 28, 209.

(e) Brown, R. C. D. Angew. Chem., Int. Ed. 2005, 44, 850

(f) Balme, G.; Bouyssi, D.; Monteiro, N. Heterocycles 2007, 73, 87.

[33] Tang, P.; Ritter, T. Tetrahedron 2011, 67, 4449.

[34] Viterisi, A.; Orsini, A.; Weibel, J.-M.; Pale, P. Tetrahedron Lett. 2006, 47, 2779.

[35] (a) Iqbal, J.; Bhatia, B.; Nayyar, N. K. Chem. Rev. 1994, 94, 519.

(b) Snider, B. B. Chem. Rev. 1996, 96, 339.

(c) Alagoz, O.; Yilmaz, M.; Tarik Pekel, A. Synth. Commun. 2006, 36, 1005.

(d) Lee, Y. R.; Byun, M. W.; Kim, B. S. Bull. Korean Chem. Soc. 1998, 19, 1080.

(e) Liu, W.; Jiang, H.; Zhang, M.; Qi, C. J. Org. Chem. 2010, 75, 966.

(f) Yan, R.; Huang, J.; Luo, J.; Wen, P.; Huang, G.; Liang, Y. Synlett 2010, 1071.

(g) Sivan, A.; Deepthi, A.; Nandialath, V. Synthesis 2011, 2466.

[36] Katritzky, A. R. Comprehensive Heterocyclic Chemistry III, Elsevier, Amsterdam, New York, 2008

[37] (a) Katritzky, A. R.; Xu, Y.-J.; Tu, H. J. Org. Chem. 2003, 68, 4935. (b) Langer, S. Z.; Arbilla, S.; Benavides, J.; Scatton, B. Adv. Biochem. Psychopharmacol. 1990, 46, 61.

(c) Carminati, G. M. Farmaco, Ed. Prat. 1978, 33, 68.

(d) Allen, J.; Parent, G.; Tizot, A. J. Labelled Compd. Radiopharm. 1986, 23, 807.

[38] (a) Groebke, K.; Weber, L.; Mehlin, F. Synlett 1998, 661.

(b) Yadav, J. S.; Reddy, B. V. S. Y.; Rao, G.; Srinivas, M.; Narsaiah, A. V. Tetrahedron Lett. 2007, 48, 7717.

(c) Bakherad, M.; Nasr-Isfahani, H.; Keivanloo, A.; Doostmohammadi, N. Tetrahedron Lett. 2008, 49, 3819.

(d) Adib, M.; Mohamadi, A.; Sheikhi, E.; Ansari, S.; Bijanzadeh, H. R. Synlett. 2010, 1606.

[39] He, C.; Hao, J.; Xu, H.; Mo, Y.; Liu, H.; Han, J.; Lei, A. Chem. Commun. 2012, 48, 11073.

[40] He, C.; Guo, S.; Ke, J.; Hao, J.; Xu, H.; Chen, H.; Lei, A. J. Am. Chem. Soc. 2012, 134, 5766.

[41] Ueda, S.; Nagasawa, H. J. Am. Chem. Soc. 2009, 131, 15080.

[42] (a) Schoenberg, A.; Bartoletti, I.; Heck, R. F. J. Org. Chem. 1974, $39,3318$.

(b) Schoenberg, A.; Heck, R. F. J. Org. Chem. 1974, 39, 3327.

[43] (a) Zhao, Y.; Jin, L.; Li, P.; Lei, A. J. Am. Chem. Soc. 2008, 130, 9429 .

(b) Ritleng, V.; Sirlin, C.; Pfeffer, M. Chem. Rev. 2002, 102, 1731.

(c) Chen, X.; Engle, K. M.; Wang, D.-H.; Yu, J.-Q. Angew. Chem., Int. Ed. 2009, 48, 5094.

(d) Lu, W.; Yamaoka, Y.; Taniguchi, Y.; Kitamura, T.; Takaki, K.; Fujiwara, Y. J. Organomet. Chem. 1999, 580, 290.

(e) Dupont, J.; Consorti, C. S.; Spencer, J. Chem. Rev. 2005, 105, 2527.

(f) Giri, R.; Yu, J.-Q. J. Am. Chem. Soc. 2008, 130, 14082.

(g) Guan, Z.-H.; Ren, Z.-H.; Spinella, S. M.; Yu, S.; Liang, Y.-M.; Zhang, X. J. Am. Chem. Soc. 2009, 131, 729.

(h) Inoue, S.; Shiota, H.; Fukumoto, Y.; Chatani, N. J. Am. Chem. Soc. 2009, 131, 6898.

(i) Yu. L. M.; Xu, Q. H.; Cai, M. Z. Chin. J. Org. Chem. 2008, 28, 256 (in Chinese).

(余腊妹, 许秋华, 蔡明中, 有机化学, 2008, 28, 256.)

[44] (a) Orito, K.; Horibata, A.; Nakamura, T.; Ushito, H.; Nagasaki, H.; Yuguchi, M.; Yamashita, S.; Tokuda, M. J. Am. Chem. Soc. 2004, 
$126,14342$.

(b) Ohashi, S.; Sakaguchi, S.; Ishii, Y. Chem. Commun. 2005, 486. (c) Haffemayer, B.; Gulias, M.; Gaunt, M. J. Chem. Sci. 2011, 2, 312 .

[45] (a) Bew, S. P. Compr. Org. Funct. Group Transform. II 2005, 5, 19. (b) Goossen, L. J.; Rodriguez, N.; Goossen, K. Angew. Chem. 2008, 120, 3144; Angew. Chem., Int. Ed. 2008, 47, 3100.

(c) Itahara, T. Chem. Lett. 1982, 1151.

(d) Itahara, T. Chem. Lett. 1983, 127.

[46] (a) Okamoto, N.; Miwa, Y.; Minami, H.; Takeda, K.; Yanada, R. Angew. Chem. 2009, 121, 9873; Angew. Chem. Int. Ed. 2009, 48, 9693.

(b) Cacchi, S.; Fabrizi, G. Chem. Rev. 2005, 105, 2873.

(c) Wolfe, J. P.; Thomas, J. S. Curr. Org. Chem. 2005, 9, 625.

(d) Joucla, L.; Djakovitch, L. Adv. Synth. Catal. 2009, 351, 673.

[47] Zhang, H.; Liu, D.; Chen, C.; Liu, C.; Lei, A. Chem. Eur. J. 2011, 17,9581 .

[48] (a) Bew, S. P. Compr. Org. Funct. Group Transform. II 2005, 5, 19. (b) Goossen, L. J.; Rodriguez, N.; Goossen, K. Angew. Chem., Int. Ed. 2008, 47, 3100.

[49] (a) Grimster, N. P.; Gauntlett, C.; Godfrey, C. R. A.; Gaunt, M. J. Angew. Chem., Int. Ed. 2005, 44, 3125.

(b) Cacchi, S.; Fabrizi, G. Chem. Rev. 2005, 105, 2873.

(c) Wolfe, J. P.; Thomas, J. S. Curr. Org. Chem. 2005, 9, 625.

(d) Stuart, D. R.; Fagnou, K. Science 2007, 316, 1172.

(e) Seregin, I. V.; Gevorgyan, V. Chem. Soc. Rev. 2007, 36, 1173.

(f) Joucla, L.; Djakovitch, L. Adv. Synth. Catal. 2009, 351, 673.

[50] (a) Stuart, D. R.; Fagnou, K. Science 2007, 316, 1172.

(b) Yeung, C. S.; Dong, V. M. Chem. Rev. 2011, 111, 1215.

(c) Liu, C.; Zhang, H.; Shi, W.; Lei, A. Chem. Rev. 2011, 111, 1780.

(d) Cho, S. H.; Kim, J. Y.; Kwak, J.; Chang, S. Chem. Soc. Rev. 2011, 40, 5068 .

[51] Liu, Q.; Zhang, H.; Lei, A. Angew. Chem., Int. Ed. 2011, 50, 10788.

[52] (a) Peres, V.; Nagem, T. J.; de Oliveira, F. F. Phytochemistry 2000, 55,683 .

(b) Pinto, M. M. M.; Sousa, M. E.; Nascimento, M. S. J. Curr. Med. Chem. 2005, 12, 2517

(c) Vieira, L. M. M.; Kijjoa, A. Curr. Med. Chem. 2005, 12, 2413.

(d) Riscoe, M.; Kelly, J. X.; Winter, R. Curr. Med. Chem. 2005, 12, 2539.

(e) El-Seedi, H. R.; El-Ghorab, D. M. H.; El-Barbary, M. A.; Zayed, M. F.; Goeransson, U.; Larsson, S.; Verpoorte, R. Curr. Med. Chem. 2009, 16, 2581.

(f) Saleem, M.; Nazir, M.; Ali, M. S.; Hussain, H.; Lee, Y. S.; Riaz, N.; Jabbar, A. Nat. Prod. Rep. 2010, 27, 238.

[53] Zhao, J.; Larock, R. C. Org. Lett. 2005, 7, 4273.

[54] Zhang, H.; Shi, R.; Gan, P.; Liu, C.; Ding, A.; Wang, Q.; Lei, A. Angew. Chem., Int. Ed. 2012, 51, 5204.

[55] Labadie, J. W.; Stille, J. K. J. Am. Chem. Soc. 1983, 105, 6129.

[56] (a) Izawa, Y.; Shimizu, I.; Yamamoto, A. Bull. Chem. Soc. Jpn. 2004, 77, 2033.

(b) Hu, Y.; Liu, J.; Lu, Z.; Luo, X.; Zhang, H.; Lan, Y.; Lei, A. J. Am. Chem. Soc. 2010, 132, 3153.

[57] Otera, J. Esterification: Methods, Reactions, and Applications, Wiley-VCH, Weinheim, 2003.

[58] (a) Schoenberg, A.; Heck, R. F. J. Org. Chem. 1974, 39, 3327.

(b) Brennfuhrer, A.; Neumann, H.; Beller, M. Angew. Chem., Int. Ed. 2009, 48, 4114.

[59] (a) Larock, R. C. Comprehensive Organic Transformations: A Guide to Functional Group Preparations, 2nd ed., Wiley-VCH, New York, 1999.

(b) Li, Q. L.; Gu, C. Z.; Yin, H.; Zhang, Y. Chin. J. Org. Chem.
2005, 25, 1416 (in Chinese).

(李前荣，顾承志，尹浩，张毅，有机化学, 2005, 25, 1416.)

[60] (a) Ekoue-Kovi, K.; Wolf, C. Chem. Eur. J. 2008, 14, 6302.

(b) Yoo, W.-J.; Li, C.-J. Tetrahedron Lett. 2007, 48, 1033.

(c) Yoo, W.-J.; Li, C.-J. J. Org. Chem. 2006, 71, 6266.

(d) Xu, B.; Liu, X.; Haubrich, J.; Friend, C. M. Nat. Chem. 2010, 2, 61.

(e) Wu, X.-F.; Darcel, C. Eur. J. Org. Chem. 2009, 1144.

[61] (a) Xu, B.; Liu, X.; Haubrich, J.; Madix, R. J.; Friend, C. M. Angew. Chem., Int. Ed. 2009, 48, 4206.

(b) Oliveira, R. L.; Kiyohara, P. K.; Rossi, L. M. Green Chem. 2009, 11, 1366.

(c) Miyamura, H.; Yasukawa, T.; Kobayashi, S. Green Chem. 2010, 12,776 .

(d) Owston, N. A.; Parker, A. J.; Williams, J. M. J. Chem. Commun. 2008, 624 .

(e) Zeng, T.; Song, G.; Li, C.-J. Chem. Commun. 2009, 6249.

(f) Zhang, J.; Gandelman, M.; Shimon, L. J. W.; Milstein, D. Dalton Trans. 2007, 107.

(g) Zhang, J.; Leitus, G.; Ben-David, Y.; Milstein, D. J. Am. Chem. Soc. 2005, 127, 10840 .

[62] Liu, C.; Wang, J.; Meng, L.; Deng, Y.; Li, Y.; Lei, A. Angew. Chem., Int. Ed. 2011, 50, 5144.

[63] Luo, X.; Zhang, H.; Duan, H.; Liu, Q.; Zhu, L.; Zhang, T.; Lei, A. Org. Lett. 2007, 9, 4571.

[64] (a) Sigman, M. S.; Jensen, D. R. Acc. Chem. Res. 2006, 39, 221.

(b) Sheldon, R. A.; Arends, I. W. C. E.; ten Brink, G.-J.; Dijksman, A. Acc. Chem. Res. 2002, 35, 774.

(c) Schultz, M. J.; Sigman, M. S. Tetrahedron 2006, 62, 8227.

(d) Stahl, S. S. Angew. Chem., Int. Ed. 2004, 43, 3400.

(e) Kroutil, W.; Mang, H.; Edegger, K.; Faber, K. Adv. Synth. Catal. 2004, 346, 125.

(f) Mallat, T.; Baiker, A. Chem. Rev. 2004, 104, 3037.

[65] (a) Swamy, K. C. K.; Kumar, N. N. B.; Balaraman, E.; Kumar, K. V. P. P. Chem. Rev. 2009, 109, 2551.

(b) Otera, J. Esterification: Methods, Reactions, and Applications, Wiley-VCH, Weinheim, 2003.

[66] Liu, C.; Tang, S.; Zheng, L.; Liu, D.; Zhang, H.; Lei, A. Angew. Chem., Int. Ed. 2012, 51, 5662.

[67] Liu, C.; Tang, S.; Lei, A. Chem. Commun. 2013, 49, 1324.

[68] Muzart, J. Tetrahedron 2003, 59, 5789.

[69] Marquard, S. L.; Hartwig, J. F. Angew. Chem., Int. Ed. 2011, 50, 7119.

[70] (a) Widenhoefer, R. A.; Zhong, H. A.; Buchwald, S. L. J. Am. Chem. Soc. 1997, 119, 6787.

(b) Zuideveld, M. A.; Kamer, P. C. J.; van Leeuwen, P. W. N. M.; Klusener, P. A. A.; Stil, H. A.; Roobeek, C. F. J. Am. Chem. Soc. 1998, 120, 7977 .

[71] (a) Massari, S.; Daelemans, D.; Barreca, M. L.; Knezevich, A.; Sabatini, S.; Cecchetti, V.; Marcello, A.; Pannecouque, C.; Tabarrini, O. J. Med. Chem. 2010, 53, 641 .

(b) Black, L. A.; Cowart, M. D.; Gfesser, G. A.; Wakefield, B. D.; Altenbach, R. J.; Liu, H.; Zhao, C.; Hsieh, G. C. WO 2009085945 , 2009 [Chem. Abstr. 2009, 151, 123980].

(c) Aiello, S.; Wells, G.; Stone, E. L.; Kadri, H.; Bazzi, R.; Bell, D. R.; Stevens, M. F. G.; Matthews, C. S.; Bradshaw, T. D.; Westwell, A. D. J. Med. Chem. 2008, 51, 5135.

[72] (a) Moghaddam, F. M.; Boeini, H. Z. Synlett 2005, 1612. (b) Bose, D. S.; Idrees, M. Tetrahedron Lett. 2007, 48, 669. (c) Bose, D. S.; Idrees, M. J. Org. Chem. 2006, 71, 8261.

[73] (a) Vera, M. D.; Pelletier, J. C. J. Comb. Chem. 2007, 9, 569. (b) Evindar, G.; Batey, R. A. J. Org. Chem. 2006, 71, 1802. 
(c) Benedi, C.; Bravo, F.; Uriz, P.; Fernandez, E.; Claver, C.; Castillon, S. Tetrahedron Lett. 2003, 44, 6073.

[74] Wang, H.; Wang, L.; Shang, J.; Li, X.; Wang, H.; Gui, J.; Lei, A. Chem. Commun. 2012, 48, 76.

[75] (a) Pozgan, F.; Dixneuf, P. H. Adv. Synth. Catal. 2009, 351, 1737. (b) Tsai, A. S.; Bergman, R. G.; Ellman, J. A. J. Am. Chem. Soc. 2008, 130, 6316.

(c) Lebrasseur, N.; Larrosa, I. J. Am. Chem. Soc. 2008, 130, 2926.

[76] Yi, H.; Liu, Q.; Liu, J.; Zeng, Z.; Yang, Y.; Lei, A. ChemSusChem 2012, 5, 2143 .

[77] (a) Baba, H.; Moriyama, K.; Togo, H. Tetrahedron Lett. 2011, 52, 4303.

(b) Kumar, V.; Sharma, A.; Sharma, M.; Sharma, U. K.; Sinha, A. K. Tetrahedron 2007, 63, 9718. (c) Feng, J.; Liang, S.; Chen, S.-Y.; Zhang, J.; Fu, S.-S.; Yu, X.-Q. Adv. Synth. Catal. 2012, 354, 1287.

[78] Yeung, C. S.; Dong, V. M. Chem. Rev. 2011, 111, 1215.

[79] (a) Li, Z.; Cao, L.; Li, C.-J. Angew. Chem., Int. Ed. 2007, 46, 6505. (b) Borduas, N.; Powell, D. A. J. Org. Chem. 2008, 73, 7822.

(c) Li, Y.-Z.; Li, B.-J.; Lu, X.-Y.; Lin, S.; Shi, Z.-J. Angew. Chem., Int. Ed. 2009, 48, 3817.

(d) Correia, C. A.; Li, C.-J. Adv. Synth. Catal. 2010, 352, 1446.

(e) Liu, X.; Zhang, Y.; Wang, L.; Fu, H.; Jiang, Y.; Zhao, Y. J. Org. Chem. 2008, 73, 6207.

(f) Wang, Z.; Zhang, Y.; Fu, H.; Jiang, Y.; Zhao, Y. Org. Lett. 2008, 10, 1863.

(g) Fan, R.; Li, W.; Pu, D.; Zhang, L. Org. Lett. 2009, 11, 1425.

[80] Liu, L.; Floreancig, P. E. Org. Lett. 2010, 12, 4686.

(Zhao, X.) 